\title{
Data Envelopment Analisys - DEA no setor elétrico brasileiro: uma proposta para validação dos resultados
}

Sérgio Luiz da Rocha Fiúza Branco Júnior

Ms. em Administração pela UFMG/CEPEAD. Professor na Fundação Dom Cabral e na Fundação Getúlio Vargas

sergio@redealumni.com

Ana Lúcia Miranda Lopes

Pós-Doutorado pela Aston Business School, Birmingham, UK. Professora Associada do Departamento de Ciências Administrativas da Faculdade de Ciências Econômicas FACE/UFMG analopes.ufmg@gmail.com

Bruno de Almeida Vilela

Doutorado sanduíche pela Technische Universität Braunschweig, no Institut für Controlling \& Unternehmensrechnung. Doutorando pelo Centro de Pesquisas e Pós-Graduação em Administração da Universidade Federal de Minas Gerais

brunoavilela@gmail.com

Reginaldo de Jesus Carvalho Lima

Doutorado em Administração pelo CEPEAD/UFMG. Professor do MPA e coordenador do curso de graduação em Administração da FPL

reginaldo.lima@fpl.edu.br

\begin{tabular}{|c|} 
Editor Científico: José Edson Lara \\
Organização Comitê Científico \\
Double Blind Review pelo SEER/OJS \\
Recebido em 27.05.2018 \\
Aprovado em 28.06.2018
\end{tabular}

\footnotetext{
(c) (9) (9)

Este trabalho foi licenciado com uma Licença Creative Commons - Atribuição - Não Comercial 3.0 Brasil
} 


\title{
Resumo
}

O presente trabalho buscou identificar o modelo de Data Envelopment Analysis (DEA) que melhor reflete a visão dos especialistas do setor de distribuição de energia elétrica brasileira a respeito da eficiência das empresas que nele atuam. DEA tem sido largamente utilizado para fins de regulação neste setor como metodologia de identificação do custo eficiente das empresas de distribuição e transmissão de energia elétrica. Para obtenção dos resultados, foram compiladas opiniões de especialistas do setor, de forma a obter um ranking das empresas de distribuição de energia elétrica a ser confrontado com diferentes modelos DEA utilizados na literatura, utilizados pela Agência Nacional de Energia Elétrica - ANEEL, e propostos em audiências públicas. Para a análise, utilizou-se ferramentas estatísticas como correlação de Spearman e o teste $t$ de Student. Os resultados apontaram que os modelos que melhor refletem a opinião dos especialistas foram os propostos por Bogetoft e Lopes (2015).

Palavras-chave: Data Envelopment Analysis - DEA, regulação de empresas de energia, distribuição de energia elétrica, validação.

\section{Data Envelopment Analisys - DEA in the brazilian electricity sector:}

\section{a proposal for validation of the results}

\begin{abstract}
The present work sought to identify the Data Envelopment Analysis (DEA) model that best reflects the view of specialists in the Brazilian electricity distribution sector regarding the efficiency of the companies that operate in it. DEA has been widely used for regulatory purposes in this sector as a methodology for identifying the cost efficiency of electricity distribution and transmission companies. In order to obtain the results, expert opinions were compiled from the sector, in order to obtain a ranking of the electricity distribution companies to be confronted with different DEA models used in the literature, used by the National Electric Energy Agency (ANEEL) and proposed in public hearings. Statistical tools such as Spearman correlation and Student's t-test were used for the analysis. The results showed that the models that best reflect the opinion of the experts were those proposed by Bogetoft and Lopes (2015).
\end{abstract}

Keywords: Data Envelopment Analysis - DEA, regulation of energy companies, distribution of electricity, validation. 


\section{Data Envelopment Analisys - DEA en el sector eléctrico brasileño: una propuesta para la validación de los resultados}

\section{Resumen}

El presente trabajo buscó identificar el modelo de análisis de envolvimiento de datos (DEA) que mejor refleja la opinión de especialistas en el sector de distribución de electricidad brasileño con respecto a la eficiencia de las empresas que operan en él. DEA se ha utilizado ampliamente con fines regulatorios en este sector como una metodología para identificar la rentabilidad de las empresas de distribución y transmisión de electricidad. Para obtener los resultados, se recopilaron opiniones de expertos del sector, con el objetivo de obtener un ranking de las empresas distribuidoras de electricidad para enfrentar los diferentes modelos DEA utilizados en la literatura, utilizados por la Agencia Nacional de Energía Eléctrica (ANEEL) y propuestos en audiencias públicas. Las herramientas estadísticas como la correlación de Spearman y la prueba t de Student se usaron para el análisis. Los resultados mostraron que los modelos que mejor reflejan la opinión de los expertos fueron los propuestos por Bogetoft y Lopes (2015).

Palabras clave: Data Envelopment Analisys - DEA, regulación de empresas de energía, distribución de electricidad, validación.

\section{Introdução}

As empresas de distribuição de energia elétrica estão espalhadas por todo o país, com o objetivo de entregar a energia produzida aos consumidores. Elas não se diferenciam apenas pela região de atuação; neste mercado existem empresas privadas, estatais e de economia mista; empresas que atendem a estados inteiros e empresas que atendem a pequenas regiões; empresas de pequeno, médio e grande porte; enfim, empresas que podem ser diferenciadas a partir de inúmeros quesitos (Pires \& Piccinini, 1999). Pelos investimentos elevados, necessários à implantação de uma nova empresa, o que impede a entrada de concorrentes, o setor de distribuição de energia elétrica é considerado monopólio natural (Agrell \& Bogetoft, 2016). As distribuidoras de energia não podem estabelecer seus próprios preços, pois são signatárias de contratos de concessão e reguladas pela Agência Nacional de Energia Elétrica - ANEEL. A Agência tem, como uma de suas responsabilidades, a de fixar as tarifas máximas cobradas do consumidor por cada empresa de distribuição de energia 
elétrica brasileira. Desta forma, sobre este setor é instituído o modelo de regulação de preços ou de regulação por incentivos (Salgado \& Motta, 2005; Jenkins \& Arriaga, 2017).

Com o objetivo de garantir o equilíbrio econômico-financeiro das distribuidoras de energia, a ANEEL - responsável por regular a produção, transmissão, distribuição e comercialização de energia elétrica no Brasil - realiza revisões das tarifas de distribuição de energia elétrica periodicamente, conforme previsto na Lei ํㅡ․ 8.987/95, tendo a primeira sido realizada em 2003 e abarcando 17 das 64 distribuidoras (Peano, 2005). Os contratos de concessão utilizam o regime de regulação price-cap, através do qual o regulador define uma tarifa inicial e o mecanismo de reajuste anual conforme inflação e ganhos de produtividade (Pessanha, Souza \& Laurencel, 2007).

Nas revisões tarifárias periódicas que ocorrem, normalmente, a cada quatro anos, o preço-teto permitido a cada empresa é definido e os custos operacionais das empresas de distribuição são avaliados via técnicas de benchmarking de forma a se identificar um valor eficiente. Os resultados são, portanto, utilizados como um componente da definição das tarifas das distribuidoras. A metodologia Data Envelopment Analysis (DEA), proposta por Charnes, Cooper e Rhodes (1978), vem sendo utilizada pelo regulador brasileiro desde 2010 e visa não apenas comparar os desempenhos de cada concessionária, como também para identificar "benchmarks hipotéticos que servem de referência para cada distribuidora". (Pessanha, Souza \& Laurencel, 2007, pg. 1).

No setor elétrico, diferentes modelagens foram propostas por Growitsch, Jamasb e Pollitt (2009), ao estudarem regulação no setor elétrico europeu; por Growitsch, Jamasb e Wetzel (2010), ao avaliarem empresas norueguesas; por Giannakis, Jamasb e Pollitt (2005), ao analisarem variáveis qualitativas em DEA na Inglaterra. Corton, Zimmerman e Phillips (2016) buscaram avaliar o aprimoramento na qualidade do serviços de distribuição de energia elétrica no Brasil. Rødseth (2017) avaliou a regulaçcão ambiental e eficiência alocativa na substituição de carvão mineral por gás natural no setor de energia elétrica dos Estados Unidos. Bobde e Tanaka (2018) propuseram uma avaliação de DEA com dois estágios e bootstraping da eficiência das empresas de distribuição de energia elétrica na Índia. Cada modelagem afeta os resultados encontrados, podendo inverter as definições de quais empresas são, realmente, eficientes e quais não são (Senra, 2007).

@@ Revista Gestão \& Tecnologia, Pedro Leopoldo, v. 18, n. 2, p. 139-171, mai./ago. 2018142 
Neste contexto, seria desejável um método estruturado para validar os resultados obtidos pelo regulador em sua análise de eficiência do custo operacional eficiente das distribuidoras de energia elétrica brasileiras, em cada ciclo de revisão tarifária. Existe uma lacuna com relação à validação dos resultados obtidos pela metodologia. Um estudo de Bougnol e Dula (2006) propôs a validação do uso de DEA como ferramenta para produzir um ranking de universidades. Neste trabalho, os autores comparam os resultados obtidos por DEA com os de um relatório publicado pela instituição The Center, da Universidade da Flórida, que utilizava metodologia distinta para avaliar a mesma base de dados. A comparação apontou para resultados bastante equivalentes, o que segundo os pesquisadores serviu para validar DEA como ferramenta adequada para criação de rankings de Universidades.

O uso de publicações de referência como base para a validação de DEA em outros segmentos - vide Bougnol e Dula (2006), no qual propõem comparar DEA com outros rankings que classificavam universidades - abriu espaço para questionamentos sobre a ausência de validação dos resultados obtidos em modelos DEA implementados pela ANEEL. Frente a esse cenário, o presente artigo busca identificar, dentre as modelagens propostas por acadêmicos e reguladores para 0 cálculo dos custos operacionais eficientes utilizando a metodologia DEA no setor de distribuição elétrica, qual delas melhor retrata a real eficiência das concessionárias brasileiras de energia, na visão dos especialistas da área.

Este artigo está dividido em Introdução, na qual se faz uma breve exposição dos fatos que levaram ao estabelecimento dos objetivos do mesmo; referencial teórico onde se revisa o setor elétrico brasileiro, o histórico de regulação e técnicas de benchmarking; metodologia, resultados e conclusões.

\section{Referencial teórico}

\subsection{Metodologias de benchmarking}

As metodologias de benchmarking baseadas em fronteira buscam identificar as melhores práticas dentre as empresas analisadas, estimando a fronteira eficiente. As principais metodologias de benchmarking utilizadas por reguladores do setor de energia são: Data Envelopment Analysis (DEA), Corrected Ordinary Least Square (COLS), e Stochastic Frontier Analysis (SFA), sendo o DEA baseado em 
programação linear, enquanto o COLS e o SFA são baseados em técnicas estatísticas (ZANINI, 2004).

Data Envelopment Analysis (DEA) é largamente utilizada para avaliação da eficiência de empresas brasileiras, não só do setor elétrico. Pode-se destacar o estudo de Mello et al. (2003), que utilizou DEA para avaliar a eficiência e os benchmarks dentre as companhias aéreas brasileiras; o estudo de Calôba e Lins (2005), que utilizou DEA para avaliar a eficiência das empresas do setor de distribuição de gás natural no Brasil; os trabalhos de Sollero e Lins (2004) e Saurin, Lopes e Costa Júnior (2010), que avaliam a eficiência de distribuidoras de energia elétrica brasileiras utilizando DEA; a pesquisa de Lopes et al. (2009), que utiliza DEA para selecionar carteiras de ações; Lobo et al. (2016) avaliaram a eficiência de hospitais universitários; Périco, Santana e Rebellato (2017) utilizaram bootstrap DEA para avaliar a eficiência dos aeroportos internacionais brasileiros; Pereira e Tavares (2017) analisaram a eficiência das regiões mais produtivas de cana de açúcar no Brasil.

A modelagem CCR, assim chamada por ter sido introduzida por Charnes, Cooper e Rhodes (1978), define um único escore de eficiência para cada Decision Making Unit (DMU), resultado este obtido diretamente dos dados sem a necessidade de uma especificação a priori de pesos e/ou delimitação explícita da forma funcional presumidas de relações entre entradas e saídas. Em um trabalho posterior, Banker, Charnes e Cooper (1984) abordam a escala de operação como parte da análise, tornando possível separar a eficiência técnica da eficiência de escala. Neste contexto, a ineficiência técnica é identificada como falha para se atingir os melhores níveis de produção possíveis e/ou uso de quantidades excessivas de insumos; enquanto que a ineficiência de escala reflete o fato de uma eventual DMU não estar operando na escala ótima.

DEA é utilizado para cumprir dois objetivos principais: construir uma fronteira eficiente a partir de dados empíricos e computar uma medida de eficiência relacionando dados observados com esta fronteira. A partir de pontos observados, que são combinações de insumos e produtos de um conjunto de unidades, estabelece a medida de eficiência como sendo a distância de um ponto à sua projeção na fronteira, como uma generalização das medidas de eficiência de Farrell (1957). Esta fronteira eficiente é usada como referência contra a qual são comparadas as relações 
insumo/produto de cada DMU. A fronteira estimada consiste na combinação de atividades de uma ou mais unidades de produção eficientes, formando uma envoltória linear sobre as demais unidades de produção observadas da amostra. As DMUs que possuírem a melhor relação "produto/insumo" serão consideradas eficientes e estarão situadas sobre essa fronteira, enquanto que as não eficientes estarão situadas numa região inferior à fronteira (Charnes, Cooper \& Rhodes,1978).

A análise DEA gera como resultado: (i) uma superfície envoltória que identifica as DMUs eficientes e ineficientes; (ii) uma medida de eficiência relativa para cada DMU; (iii) uma projeção da DMU ineficiente sobre a fronteira, gerando uma meta para a mesma; (iv) um conjunto-referência - benchmarks (unidades específicas que podem servir de exemplo para as DMUs ineficientes).

Um dos requisitos para a aplicação de DEA é a homogeneidade das DMUs, isto é, devem ter em comum a utilização dos mesmos inputs e outputs, realizarem as mesmas tarefas, com os mesmos objetivos, trabalhar nas mesmas condições de mercado e ter autonomia na tomada de decisões (Lins et al., 2007; Cook et al., 2017).

As diferentes em formulações DEA diferem, fundamentalmente: quanto ao tipo de orientação e quanto a suposições sobre o retorno de escala exibido, conforme tecnologia de produção. Com relação a este último item, cada modelagem DEA deve assumir um tipo de retorno de escala que represente o comportamento da operação à medida que os níveis de produção são significativamente alterados para mais ou para menos. Os tipos de retornos de escala geralmente considerados são os retornos constantes de escala (constant returns to scale - CRS), retornos não crescentes de escala (non-increasing returns to scale - NIRS), retornos variáveis de escala (variable returns to scale - VRS) e retornos não decrescentes de escala (non-decreasing returns to scale - NDRS).

As modelagens tradicionais de DEA (CCR e BCC) são, obrigatoriamente, orientadas à contração de insumos ou expansão radial de produtos. No modelo orientado a insumos, os escores de eficiência variam entre 0 e 1 , sendo 10 valor obtido pelas DMUs eficientes. As demais DMUs devem reduzir de forma equiproporcional seus insumos, multiplicando-os pelo escore obtido, para se tornarem eficientes, ou seja, produzir as mesmas quantidades de produtos ou serviços consumindo menores quantidades de recursos. No modelo orientado a produtos, os escores são números maiores ou iguais a 1 , sendo 1 para as DMUs eficientes. As 
demais DMUs devem expandir de forma equiproporcional seus produtos, multiplicando-os pelo escore obtido, para se tornarem eficientes, ou seja, produzir mais mantendo o mesmo consumo de recursos. Ao somar-se as folgas de insumos e produtos obtém-se a eficiência de Pareto (Charnes et al., 1985)

O paper pioneiro de DEA, publicado em 1978 (Charnes, Cooper \& Rhodes, 1978), propõe uma avaliação da eficiência relativa de Decision Making Units (DMUs) que operem globalmente sob retornos constantes à escala (CRS) e está baseado no modelo matemático que segue.

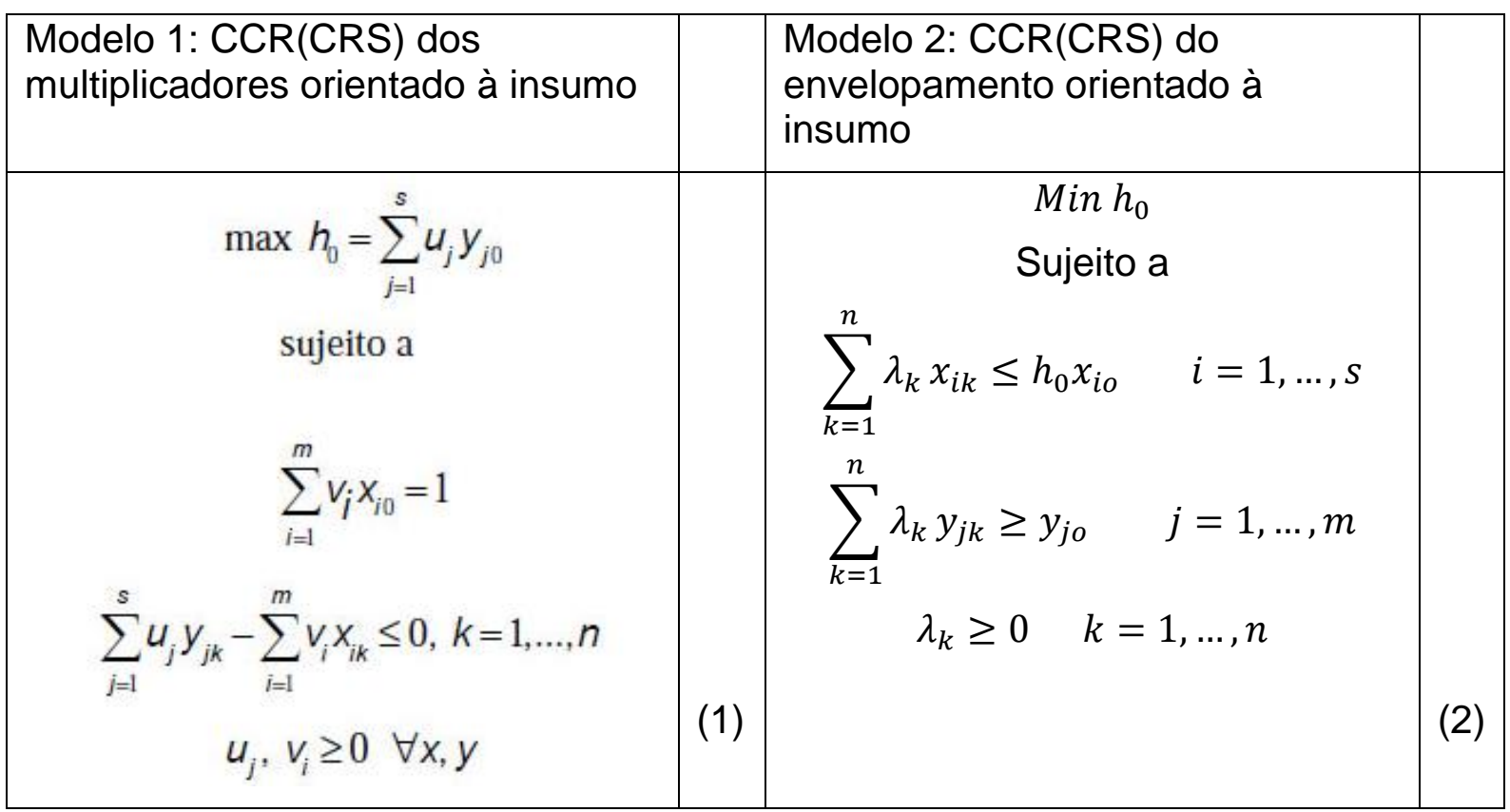

No Modelo 1 tem-se que $x_{i k}$ e $y_{j k}$ são as quantidades consumidas do recurso (input) i e as quantidades produzidas do produto (output) j pela DMU k. Os pesos vi e uj são os pesos obtidos pelo modelo de otimização de forma a maximizar ho, que corresponde à eficiência da DMU. O modelo deve ser calculado para cada DMU, alterando $x_{0}$ e yo para se obter ho específico (Charnes, Cooper \& Rhodes, 1978).

O Modelo 2 representa o dual do Modelo (1), $x_{i k}$ e $y_{j k}$ são os inputs e outputs da $D M U ~ k ; \lambda_{k}$ são as contribuições da DMU k na projeção eficiente da $\mathrm{DMU}_{0}$. Por meio da adição de uma restrição em que o somatório dos lambdas seja igual a $1\left(\sum_{j=1}^{n} \lambda_{j}=1\right)$ ao Modelo 2 ou de uma constante $\mu_{0}$ ao Modelo 1. Banker, Charnes e Cooper (1984) expandiram a aplicação da metodologia DEA para utilização em grupos de empresas que operam sob diferentes retornos de escala (crescente, constante e decrescente). 
Este modelo foi chamado de VRS (variable returns to scale) ou BCC (Banker, Charnes e Cooper).

\subsection{Setor Elétrico Brasileiro}

O segmento de distribuição é aquele que recebe grande quantidade de energia do sistema de transmissão e a distribui de forma pulverizada para consumidores médios e pequenos (Lugoboni, Zittei \& Paulino, 2014). Existem também unidades geradoras de menor porte, normalmente menores do que $30 \mathrm{MW}$, que injetam sua produção nas redes do sistema de distribuição. No Brasil, o segmento de distribuição é composto por 63 concessionárias, as quais são responsáveis pela administração e operação de linhas de distribuição de menor tensão (abaixo de 230 mil Volts), mas principalmente das redes de média e baixa tensão, como aquelas instaladas nas ruas e avenidas das grandes cidades (Péres, Campos \& Liang, 2015). É a empresa distribuidora quem faz com que a energia elétrica chegue às residências e pequenos comércios e indústrias.

As empresas de transmissão e distribuição de energia elétrica Brasileiras tem seus preços regulados pela Agência Nacional de Energia Elétrica - ANEEL, que é a agência reguladora do setor. Desse modo, essas empresas não são livres para praticar os preços que desejam, inserindo-se no contexto dos contratos de concessão, que usualmente contam com mecanismos de revisões e reajustes tarifários periódicos, operacionalizados pela própria agência reguladora (Associação Brasileira de Distribuidores de Energia Elétrica').

\subsubsection{Regulação do Setor de Distribuição de Energia Elétrica e os ciclos tarifários}

Como órgão regulador do setor, a ANEEL é responsável por decisões importantes para as empresas de distribuição de energia elétrica. Dentre elas, podese destacar o controle das empresas que podem entrar ou sair do setor, fixação dos valores máximos para as tarifas cobradas ao cosumidor, metas de qualidade e universalização dos serviços e participação máxima no mercado nacional ou regional.

\footnotetext{
${ }^{1}$ Informação disponível em: http://www.abradee.com.br/setor-de-distribuicao/a-distribuicao-de-energia
} 
A distribuição de energia, por suas características físicas e estruturais, constitui um monopólio natural. Dessa forma, qualquer tipo de concorrência nesse mercado é fraco ou inexistente. Estas condições podem prejudicar o consumidor não só pelos preços abusivos, mas pela ausência ou insuficiência no fornecimento de energia para todos os habitantes, principalmente para aqueles situados em áreas de difícil acesso ou distantes de centros urbanos.

Com o objetivo de garantir o equilíbrio econômico-financeiro dos serviços das distribuidoras de energia, o regulador revisa as tarifas permitidas periodicamente, conforme previsto na Lei no 8.987/95. As revisões tarifárias são processos de reajuste dos valores das tarifas cobradas aos consumidores pelas empresas concessionárias de distribuição de energia. Essas tarifas podem ser aumentadas ou reduzidas de acordo com os resultados provenientes dessas revisões, que irão avaliar as mudanças ocorridas nos custos e no mercado das empresas, na eficiência das mesmas, com o objetivo de garantir tarifas mais justas aos consumidores e retornos adequados aos empresários (LMDM Consultoria, 2013).

O processo de revisão tarifária periódica é composto pelo cálculo da Parcela $A$, Parcela $B$ e fator $X$, que, quando unificados compõem a tarifa, além do estabelecimento de metas de qualidade e perdas não técnicas. A parcela $A$ aborda custos não gerenciáveis como compra de energia, encargos setoriais e transporte de energia. São denominados não gerenciáveis por não dependerem diretamente da administração das distribuidoras. (Peano, 2005)

A parcela $B$ compõe os custos gerenciáveis: remuneração de capital, custos operacionais e taxas. O custo operacional, foco do presente artigo, é o custo total empregado pela empresa para o serviço de distribuição de energia, no qual estão incluídos custos de "recursos humanos (administrativo e de operação e manutenção), de infraestrutura física (edificações, móveis, sistema de informática), de materiais e serviços", conforme a Nota Técnica n 407/2014 (Agência Nacional De Energia Elétrica, 2014).

Os ciclos tarifários duram entre quatro e cinco anos e, ao fim desse prazo, a ANEEL avalia os dados realizados das concessionárias, dando início a um novo ciclo de revisão tarifária (Siciliano, 2005). O primeiro, segundo, terceiro e quarto ciclos foram iniciados, respectivamente, em 2003, 2007, 2011 e 2015, respectivamente denominados de 1CRTP, 2CRTP, 3CRTP e 4CRTP. 
No cálculo da parcela $B$, é necessário determinar o custo operacional permitido para cada distribuidora. A regulação prevê a identificação do custo operacional eficiente, isto é, o custo que cada distribuidora efetivamente teria para atender seu mercado, caso sua gestão de recursos fosse ótima. Existe uma gama de alternativas possíveis para se medir eficiência e uma série de escolhas que vão desde a ferramenta ou método a ser utilizado até a especificação de parâmetros, forma de construção de variáveis, tratamento dos dados, análise de outliers entre outros (Peano, 2005).

O objetivo deste artigo é o de discutir e validar modelos DEA utilizando dados do $4^{\circ}$ ciclo tarifário (4CRTP), publicados em 6 de junho de 2014, como parte da Audiência Pública No. 023/2014. Para tal, serão elencadas as principais características relacionadas à avaliação de eficiência dos três ciclos anteriores.

No quarto ciclo de revisão tarifária periódica (4CRTP), iniciado na Consulta Pública n 23/2014, a ANEEL optou pela utilização de DEA como única metodologia de benchmarking para a definição do custo operacional eficiente (Nota Técnica no 192/2014 - SRE/ANEEL). A variável de insumo utilizada foi o custo operacional, mensurado pelos gastos com pessoal, materiais, serviços de terceiros e outros custos contabilmente associados à operação (Nota Técnica no 192/2014-SRE/ANEEL). Considerando que o custo da mão de obra no Brasil varia entre as Regiões, o custo de pessoal foi ajustado pelas diferenças salariais de cada região aonde a empresa localiza sua sede.

A Nota Técnica no 192/2014 - SRE/ANEEL coloca em discussão seis diferentes modelos DEA, enquanto mantém a divisão das empresas em 2 grupos, conforme o 3CRTP. Além disto, propõe que os dados utilizados para o cálculo dos escores de eficiência sejam dados em painel dos anos 2010 a 2012.

Com relação aos produtos, o 4CRTP trouxe mudanças significativas propondo, em seus seis modelos, as variáveis de extensão de rede (total ou dividida entre rede alta e rede de distribuição), mercado ponderado ou MVA instalado, número de consumidores (total ou dividido entre urbanos e rurais), todos estes como produtos positivos; perdas não técnicas e qualidade, nesta NT representada por Energia Não Distribuida (ENDIST), estes como produtos negativos. 
A Nota Técnica no 407/2014, publicada após a avaliação das contribuições dos agentes, trouxe a decisão da mudança da utilização de dados em painel pela média dos anos de 2011, 2012 e 2013. Também houveram mudanças nas variáveis de produto. Rede foi pela primeira vez separada em três categorias: rede de alta tensão, rede de distribuição aérea e rede de distribuição subterrânea. Com relação à qualidade, sua forma de cálculo foi alterada, deixando de ser Energia Não Distribuída - ENDIST - e passando a ser Consumidor Hora Interrompido - CHI. Também houve modificações no cálculo de perdas. A Figura 1 apresenta as variáveis constantes na NT 407/2014.

\section{Figura 1}

Variáveis utilizadas no primeiro estágio de DEA para definição de custos operacionais do 4CRTP

\begin{tabular}{|c|c|c|}
\hline Dimensão & Variável & Unidade \\
\hline \multirow[t]{3}{*}{ Rede } & Rede subterrânea & \multirow[t]{3}{*}{$\mathrm{Km}$} \\
\hline & Rede de distribuição aérea & \\
\hline & Rede de alta tensão & \\
\hline Consumidor & total de consumidores & unid. \\
\hline Mercado & Mercado ponderado & MWh \\
\hline Perdas & Perdas não técnicas & MWh \\
\hline Qualidade & $\begin{array}{l}\text { Consumidor Hora } \\
\text { interrompido }(\mathrm{CHI})\end{array}$ & $\mathrm{h}$ \\
\hline
\end{tabular}

Fonte: Nota Técnica ํㅜㄴ07/2014

Por fim, a NT 407/2014 deu continuidade ao que foi proposto pela NT 192/2014 no que diz respeito ao uso de restrição aos pesos - nos moldes de trade-off entre variáveis - com a adição de restrições para as variáveis Rede Subterrânea e Consumidor Hora interrompido. Com isso, o modelo final apresentou restrições aos pesos envolvendo as seguintes variáveis: opex e rede de distribuição; rede subterrânea e rede convencional; rede aérea e rede convencional; opex e consumidores; opex e potência entregue; opex e perdas não técnicas; opex e interrupções.

Com relação à prática de dividir as empresas em grupos homogêneos conforme o porte, que vigorou no 3 o CRTP, a ANEEL optou por considerar um único cluster com todas as distribuidoras. Segundo a ANEEL, o próprio regime de escala NDRS já 
controla a presença de economia de escala no setor, dado que DEA já prevê inserir empresas de múltiplos portes em seus modelos, desde que o regime esteja bem definido (Nota Técnica ํㅜ 407/2014).

Com relação à amostra utilizada, houve uma definição distinta sobre a forma de se aproveitar os dados das empresas. Com o uso de um único cluster, a configuração utilizando apenas um dado por empresa passou a ser robusta. Desta forma, a ANEEL optou por utilizar como única observação por empresa os registros médios de cada uma no triênio supracitado (Nota Técnica ํo 407/2014).

Novamente, a ANEEL manifestou não ter encontrado variáveis adequadas para satisfazer os critérios necessários para justificar a utilização de um segundo estágio em DEA. Para validar os resultados do primeiro estágio, foram utilizadas técnicas de bootstrap (Efron \& Tibshirani, 1994) e definidos intervalos de confiança para a eficiência de cada empresa. Na sequência, a ANEEL propôs algumas metodologias que poderiam utilizar os intervalos de confiança no cálculo tarifário (Nota Técnica no 407/2014).

A Nota Técnica ํㅡ 66/2015, de 24 de abril de 2015, apresentou a proposta final para a metodologia de custos operacionais, resultado da análise e consolidação das contribuições enviadas pelas distribuidoras ao longo das etapas de debate público.

\section{Metodologia}

Esta pesquisa foi dividida em cinco etapas, de modo a permitir a obtenção e análise dos dados coletados. A primeira etapa envolveu a seleção de modelos (insumos, produtos e retornos de escala) para compor a análise. Primeiramente foram elencados os modelos mais utilizados para avaliação das empresas do setor de distribuição elétrica no Brasil e no mundo. Dessa forma, os modelos implementados pela ANEEL nos dois últimos Ciclos de Revisão Tarifária Periódicos (3CRTP e 4CRTP) foram utilizados, além das modelagens propostas por autores e reguladores internacionais (Finlândia, Dinamarca, entre outros países). Também foram selecionadas algumas modelagens apresentadas por especialistas do setor $\mathrm{e}$ especialistas na metodologia DEA, como propostas de melhorias no modelo vigente. Adicionalmente, foram também analisados os modelos desenvolvidos por Bogetoft $\mathrm{e}$ Lopes (2015). A segunda etapa consistiu na coleta dos dados necessários referentes a cada concessionária de distribuição de energia no Brasil. Por norma da própria 
ANEEL, esses dados são de acesso público e são disponibilizados no site da ANEEL. Foi escolha do autor trabalhar cada modelo com o banco de dados do $4^{\circ}$. Ciclo de Revisão Tarifária Periódica, pois este era o mais atual. Neste trabalho, o escopo da análise se restringiu às concessionárias pertencentes ao Grupo 1 da ANEEL, conforme definido na Nota Técnica ํㅡ 294/2011.

A terceira etapa correspondeu à aplicação da metodologia Data Envelopment Analysis simulando diferentes modelagens. Cada distribuidora foi analisada por 45 modelos diferentes, gerando 45 escores.

A quarta etapa consistiu em coleta de dados retratando a visão de especialistas sobre a eficiência dos players do setor de distribuição de energia. Um total de 15 especialistas da área de distribuição de energia foram selecionados e responderam a um questionário do tipo Likert de 10 pontos. Neste questionário constavam os nomes das empresas a serem avaliadas e um campo para o especialista definir o ranking de eficiência que julgava correto para o setor. Dessa forma, o entrevistado preencheu o valor correspondente ao ranking da empresa, onde 1 é dado para a empresa mais ineficiente e e 10 para a mais eficiente. A pesquisa foi composta por dois grupos de especialistas. O primeiro deles foi composto por funcionários que formam o corpo técnico das empresas de distribuição de energia elétrica, enquanto o segundo foi composto por profissionais que atuam na área de modelagem e por consultores que não tinham vínculo com nenhuma empresa de distribuição.

Por fim, na quinta etapa, foi utilizada a triangulação metodológica para avaliar a correlação entre os resultados obtidos na aplicação dos diversos modelos DEA e a avaliação dos especialistas quanto à eficiência das empresas. A aplicação dessa técnica, tratada por esta pesquisa como validação convergente, teve o objetivo de avaliar qual dos modelos DEA mais se assemelhou ao resultado obtido pela avaliação dos especialistas do setor de energia elétrica. Nesta etapa também foram analisados: a sensibilidade da conclusão supracitada tendo em vista os diferentes tipos de especialistas analisados; a evolução dos modelos utilizados pela ANEEL ao longo das notas técnicas, na medida em que foram comparadas com a visão dos especialistas e a validação de tendênci as do uso de insumos, produtos e definições de retorno em escala. Os 45 modelos utilizados para calcular os escores de eficiência das distribuidoras validando-os ou não com a visão dos especialistas são mostrados no Apêndice I.

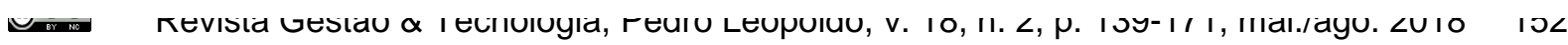




\section{Resultados}

\subsection{Resultados das Simulações}

A

Tabela 1 apresenta o resultado da aplicação de DEA nos 45 modelos analisados neste trabalho. Os modelos foram ordenados por média dos escores de eficiência, decrescente, da esquerda para a direita. As DMUs foram ordenadas também por média dos escores de eficiência, descrescente, de cima para baixo.

De forma a analisar semelhanças e diferenças entre os modelos, foram realizadas diferentes análises presentes nas Tabelas 2 e 3. A Tabela 2 apresenta a correlação de Spearman entre os resultados dos modelos. Os modelos foram reordenados conforme a média das correlações de cada um com os demais. 


\section{Tabela 1}

Resultado das aplicações de DEA

Gapresss

PRATNNGA $1001001001001001001001001001001001001001001001009610010096941001009594979494949410010094949594 \quad 83 \quad 82 \quad 7996 \quad 9694 \quad 859495 \quad 96$

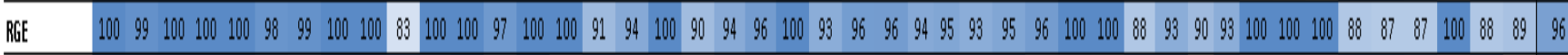

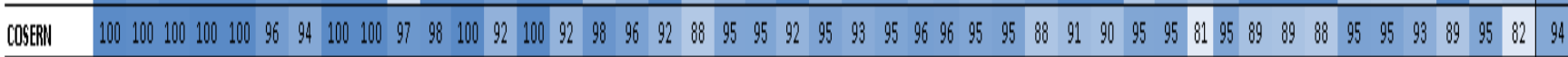

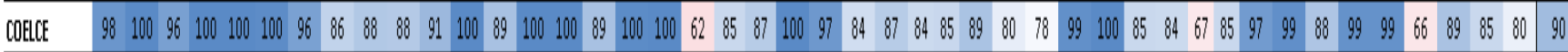

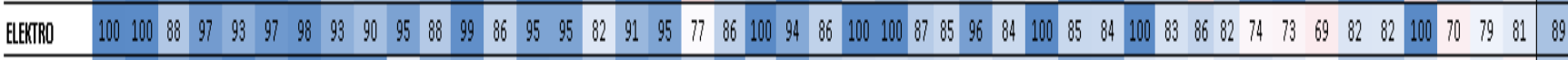

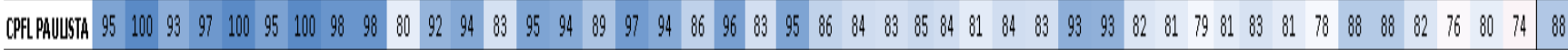

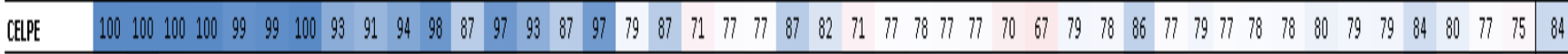

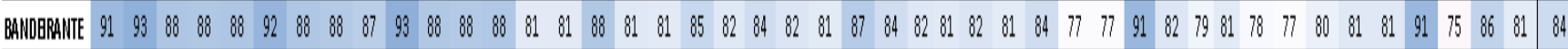
\begin{tabular}{llllllllllllllllllllllllllllllllllllllllllllllll}
\hline COEEBA & 86 & 100 & 87 & 88 & 87 & 92 & 99 & 74 & 76 & 85 & 77 & 99 & 77 & 100 & 98 & 77 & 86 & 97 & 52 & 94 & 79 & 97 & 86 & 80 & 79 & 79 & 79 & 79 & 79 & 69 & 89 & 89 & 77 & 79 & 79 & 79 & 89 & 89 & 87 & 83 & 83 & 67 & 87 & 77 & 79 & 84
\end{tabular}

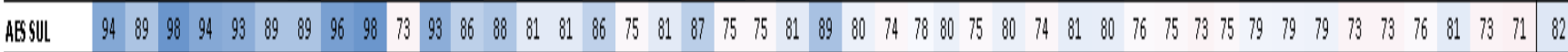

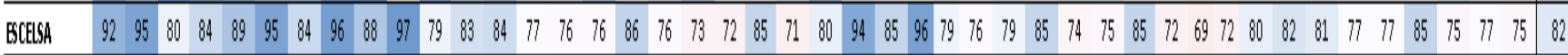

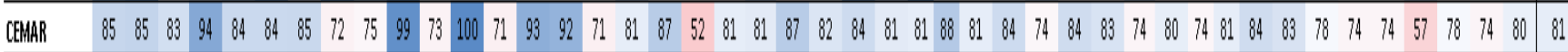

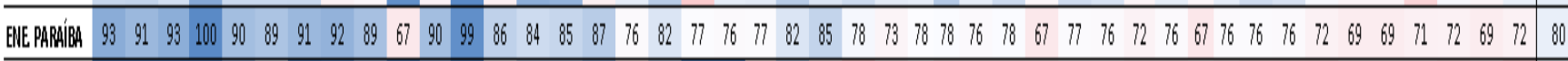

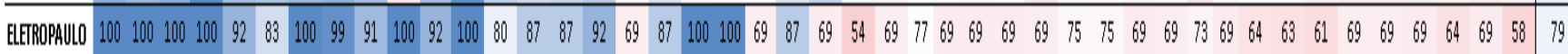

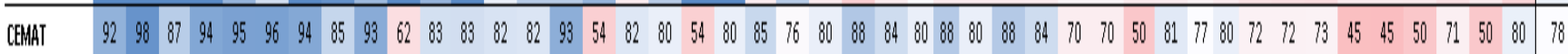

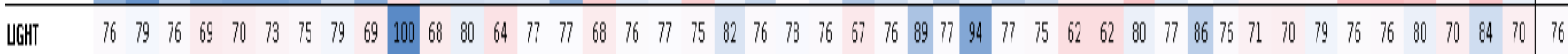

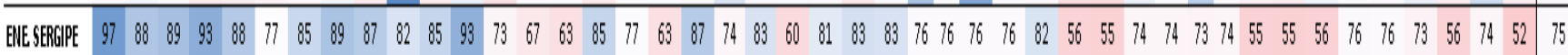

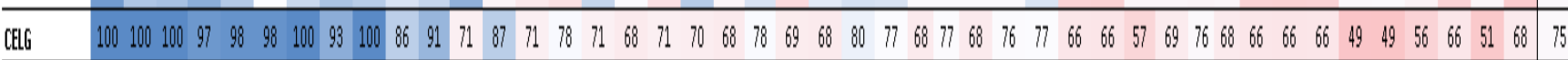

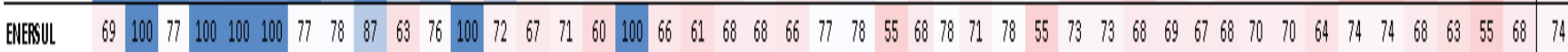

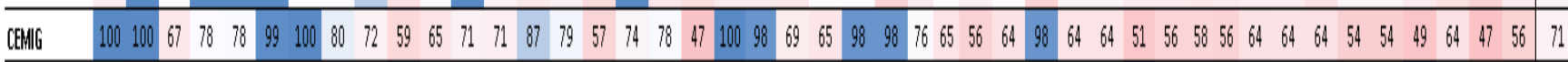

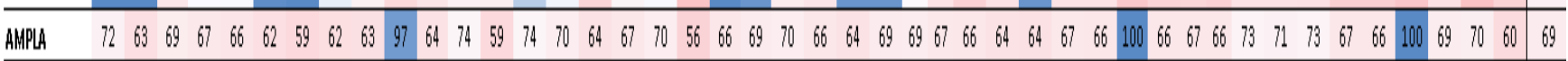

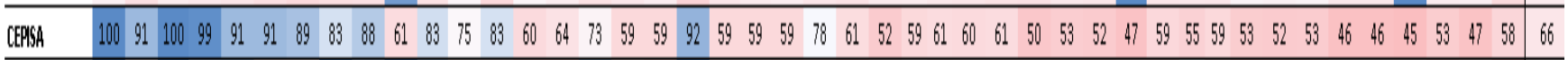

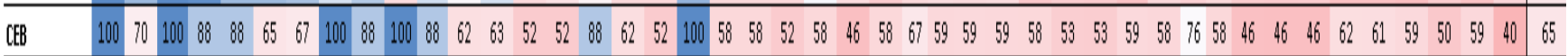

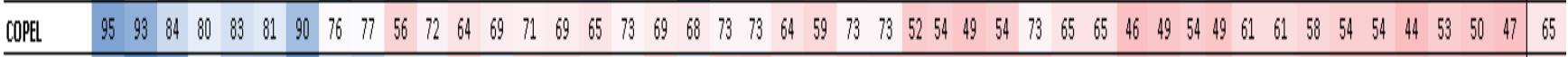

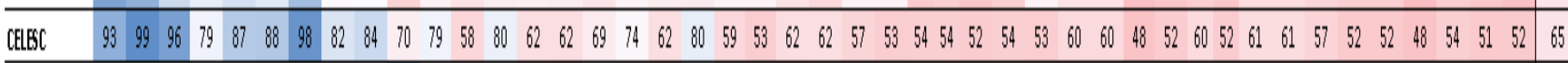

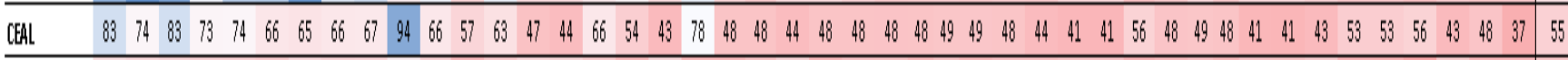

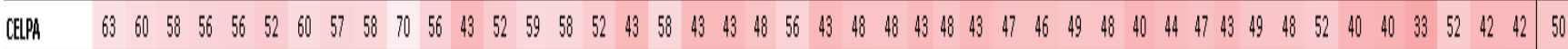

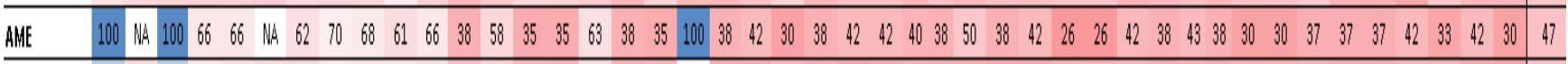

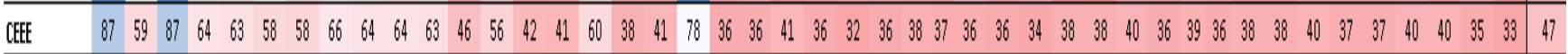

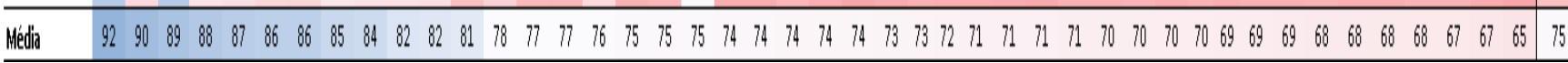

Observações: (1) Resultados multiplicados por 100; (2) Tonalidade azul ressalta valores próximos de 100; (3) Tonalidade vermelha ressalta valores próximos de 25; (4) Tonalidade branca mostra valores intermediários. 


\section{Tabela 2}

\section{Correlação entre modelos DEA}

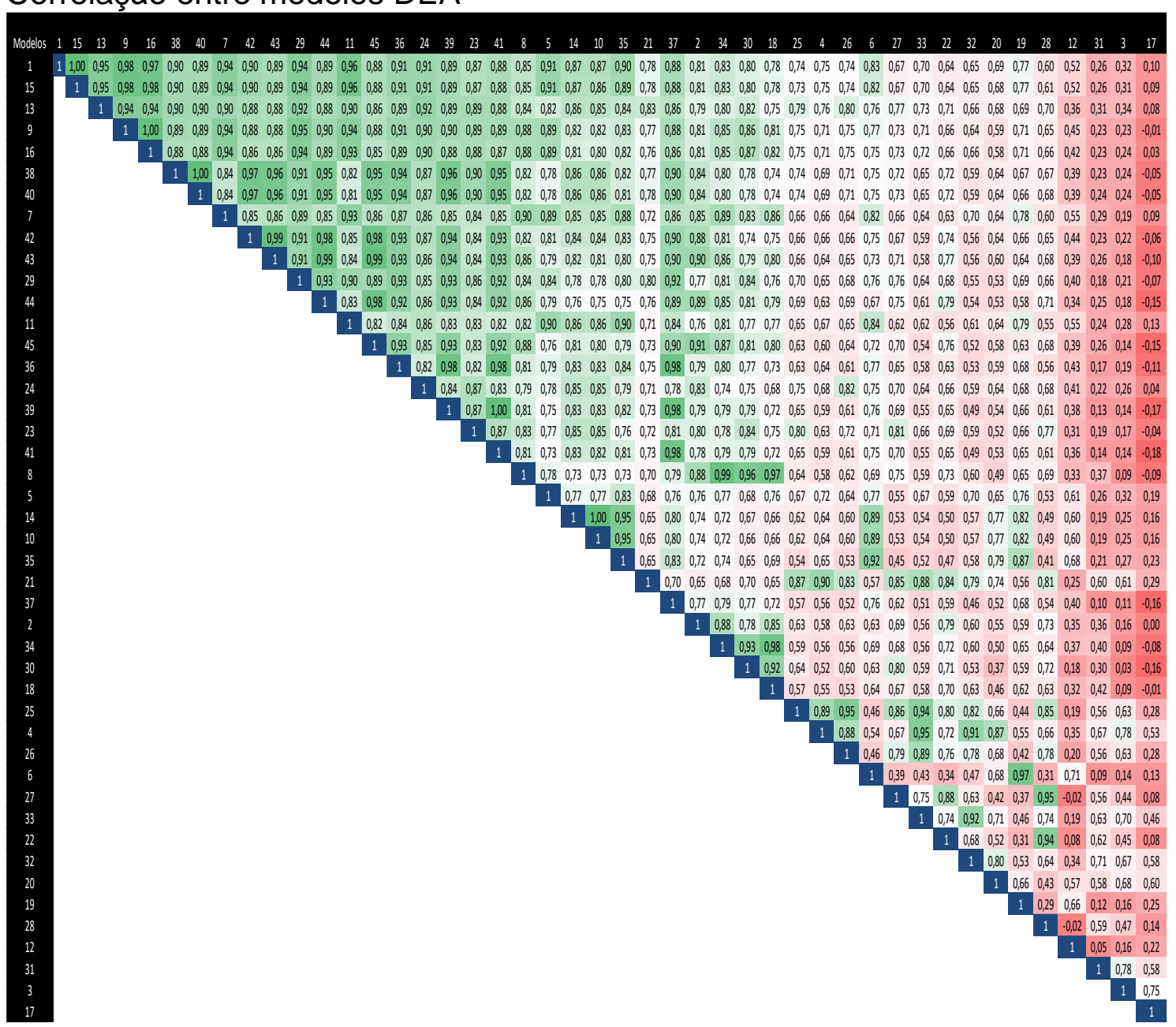

Observações: (1) Tonalidade verde ressalta valores próximos de 1. (2) Tonalidade vermelha ressalta valores próximos de $-0,18$ (menor valor). (3) Tonalidade branca mostra valores intermediários a estes extremos. 
Tabela 3 apresenta a análise descritiva associada aos dados da Tabela 2.

Tabela 3

Análise Descritiva das Correlações entre os Modelos

\begin{tabular}{|c|c|c|c|c|c|c|c|}
\hline Modelos & Correlação Média & DesvPad Correlações & Correlação Mínima & Modelos & Correlação Média & DesvPad Correlações & Correlação Mínima \\
\hline 1 & 0,80 & 0,19 & 0,10 & 35 & 0,72 & 0,19 & 0,21 \\
\hline 15 & 0,79 & 0,19 & 0,09 & 21 & 0,72 & 0,13 & 0,25 \\
\hline 13 & 0,79 & 0,18 & 0,08 & 37 & 0,72 & 0,25 & $-0,16$ \\
\hline 9 & 0,78 & 0,21 & $-0,01$ & 2 & 0,72 & 0,19 & 0,00 \\
\hline 16 & 0,78 & 0,20 & 0,03 & 34 & 0,72 & 0,21 & $-0,08$ \\
\hline 38 & 0,77 & 0,21 & $-0,05$ & 30 & 0,69 & 0,23 & $-0,16$ \\
\hline 40 & 0,77 & 0,21 & $-0,05$ & 18 & 0,68 & 0,20 & $-0,01$ \\
\hline 7 & 0,77 & 0,19 & 0,09 & 25 & 0,68 & 0,16 & 0,19 \\
\hline 42 & 0,77 & 0,22 & $-0,06$ & 4 & 0,68 & 0,13 & 0,35 \\
\hline 43 & 0,77 & 0,22 & $-0,10$ & 26 & 0,66 & 0,15 & 0,20 \\
\hline 29 & 0,76 & 0,22 & $-0,07$ & 6 & 0,66 & 0,21 & 0,09 \\
\hline 44 & 0,76 & 0,23 & $-0,15$ & 27 & 0,65 & 0,19 & $-0,02$ \\
\hline 11 & 0,76 & 0,19 & 0,13 & 33 & 0,65 & 0,15 & 0,19 \\
\hline 45 & 0,75 & 0,23 & $-0,15$ & 22 & 0,64 & 0,18 & 0,08 \\
\hline 36 & 0,75 & 0,23 & $-0,11$ & 32 & 0,63 & 0,13 & 0,34 \\
\hline 24 & 0,75 & 0,19 & 0,04 & 20 & 0,62 & 0,12 & 0,37 \\
\hline 39 & 0,75 & 0,24 & $-0,17$ & 19 & 0,62 & 0,19 & 0,12 \\
\hline 23 & 0,74 & 0,21 & $-0,04$ & 28 & 0,62 & 0,19 & $-0,02$ \\
\hline 41 & 0,74 & 0,24 & $\begin{array}{r}-0,18 \\
\end{array}$ & 12 & 0,39 & 0,20 & $-0,02$ \\
\hline 8 & 0,73 & 0,22 & $-0,09$ & 31 & 0,35 & 0,22 & 0,05 \\
\hline 5 & 0,73 & 0,16 & 0,19 & 3 & 0,33 & 0,24 & 0,03 \\
\hline 14 & 0,73 & 0,19 & 0,16 & 17 & 0,13 & 0,27 & $-0,18$ \\
\hline 10 & 0,73 & 0,19 & 0,16 & & & & \\
\hline
\end{tabular}

A Tabela 3 sugere que há grande semelhança entre muitos dos modelos, com as poucas exceções sendo os modelos 3, 12, 17 e 31. Exceções à parte, em termos de correlação, há indícios de que os escores variam no mesmo sentido, ou seja, os modelos classificam as empresas comparativamente de forma semelhante. Isso reforça a robustez do método DEA como importante ferramenta de benchmarking. Contudo, não se pode concluir que dois modelos com alta correlação sejam equivalentes. A

Tabela 1 revela um dos principais pontos de disparidade entre os modelos. Enquanto alguns deles atribuem às empresas uma eficiência elevada, como o modelo 28; outros atribuem às mesmas empresas uma eficiência baixa, como o modelo 29.

Cabe ressaltar que a correlação entre estes dois modelos é de 0,66 , conforme a Tabela 2. Alguns pesquisadores apontam correlações superiores a 0,50 como moderadas - Mukaka (2012) - ou fortes Cohen (1992). O que chama a atenção neste caso é que um modelo pode ser avaliado (i) pelo ordenamento das DMUs em termos de eficiência, (ii) pela distância relativa entre as DMUs em termos de eficiência e (iii) $@$ 
pela média de eficiência das empresas do setor. Embora a análise de correlação seja rica para avaliar os itens (i) e (ii), o (iii) talvez seja o de maior relevância prática para as DMUs, especialmente em se tratando de regulação em um setor econômico que movimenta grandes cifras.

Foi utilizado o Teste-t pareado, que avalia a hipótese nula de que duas amostras tenham como origem duas populações com a mesma média. Esse teste comparou apenas o modelo da NT no 407/2014 da ANEEL (modelo 42) com os modelos estudados de Bogetoft e Lopes (2015) (modelos 43, 44 e 45). Os resultados se encontram na Tabela 4.

\begin{tabular}{lll}
$\begin{array}{l}\text { Tabela } 4 \\
\text { Resultado do teste-t }\end{array}$ & \\
\hline Modelo 1 & Modelo 2 & p-valor \\
\hline Aneel NT 407/2014 & Bogetoft e Lopes* Modelo B & 0,0187 \\
\hline Aneel NT 407/2014 & Bogetoft e Lopes* Modelo C & 0,0024 \\
\hline Aneel NT 407/2014 & Bogetoft e Lopes* Modelo D & 0,0003 \\
\hline
\end{tabular}

Fonte: Bogetoft e Lopes (2015)

Todos os testes- $t$ aplicados tiveram $p$-valores inferiores ao limite de 0,05 (5\%), definido como limite crítico para validar a hipótese nula, o que a rejeita. Assim sendo, para cada caso, as duas populações em questão não tem como origem populações com mesma média. Em outras palavras, por mais que possa haver forte correlação entre os modelos, os valores médios de eficiência atribuídos para as DMUs demonstram que as análises da ANEEL e a proposta em Bogetoft e Lopes (2015) tem entendimento diferente no que diz respeito à real eficiência do setor como um todo. Em geral, a média do modelo sugerido em Bogetoft e Lopes (2015) é superior à média do modelo proposto pela Aneel na Nota Técnica 407/2014.

\subsection{Resultados da pesquisa com especialistas}

A Figura mostra a distribuição de frequência dos resultados dos Modelos DEA, em contraste com a das respostas dos especialistas para as DMUs em escala comparável. 


\section{Figura 2}

Distribuição de frequência dos modelos vs das respostas do questionário
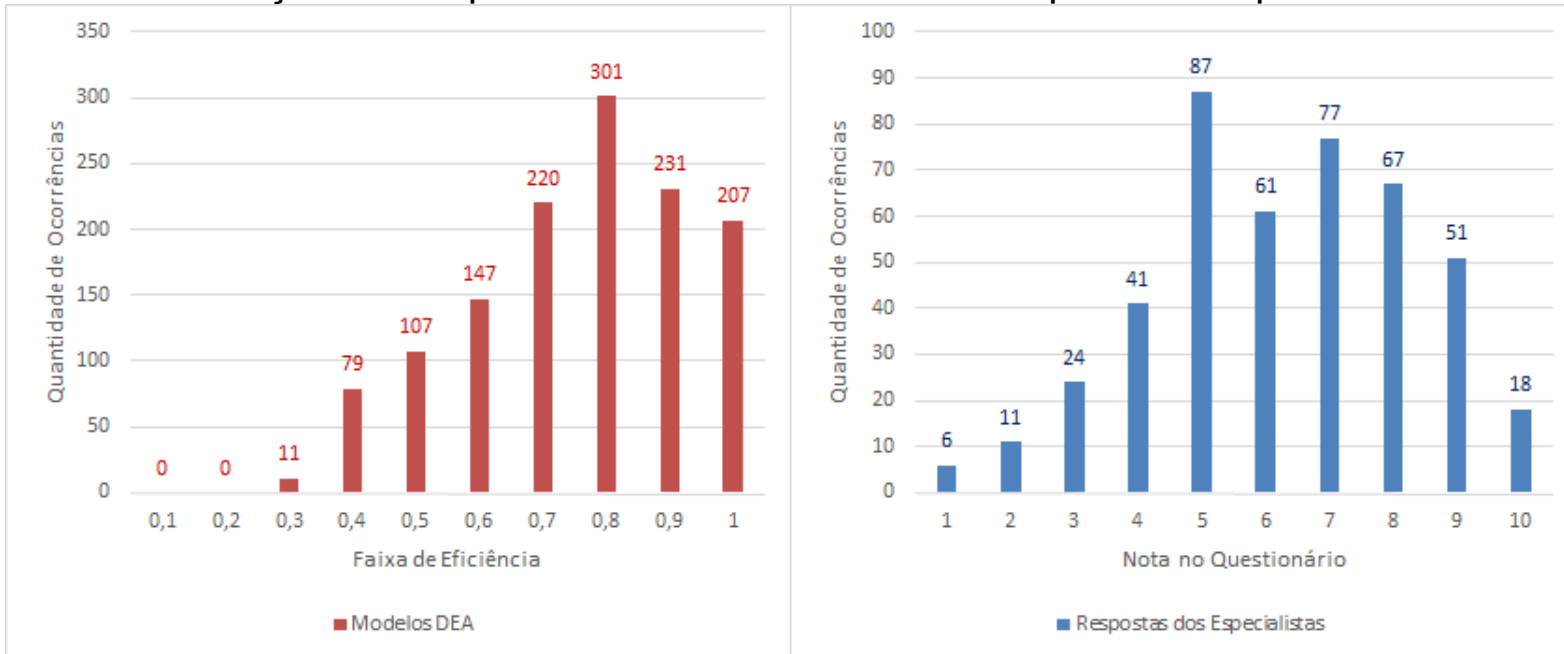

Observações: (1) Resultados arredondados para 1 casa decimal. (2) Contagem de ocorrências na população, portanto valores são absolutos.

Por se tratar de 15 especialistas e 45 modelos, as amplitudes das séries dos gráficos da

Figura não podem ser comparadas entre si. Entretanto, o formato das curvas revela que os especialistas tendem a espalhar as respostas ao longo das possibilidades, atribuindo valores muito baixos às empresas menos eficientes, e conferindo apenas às mais eficientes de uma forma global os valores mais altos. A modelagem DEA, por outro lado, tenta atribuir a cada DMU um conjunto de pesos que maximize seu resultado, o que torna relativamente frequente encontrar DMUs eficientes e raro encontrar DMUs com escore inferior a 0,2. A Tabela 5 mostra o resultado da pesquisa com os especialistas. 


\section{Tabela 5}

Resultado da Pesquisa com Especialistas

\begin{tabular}{|c|c|c|c|c|c|c|c|c|}
\hline Código & Nome & Média Especialistas & Desvio Padrão & Coeficiente de Variação & Média Distribuidoras & Média Consultores & Dif Dis: & Consult. \\
\hline D62 & RGE & 8,36 & 2,06 & $25 \%$ & 8,22 & 8,60 & $-0,38$ & \\
\hline D36 & CPFL PAULISTA & 8,33 & 1,23 & $15 \%$ & 8,13 & 8,75 & $-0,63$ & \\
\hline D35 & PIRATININGA & 7,85 & 2,34 & $30 \%$ & 7,63 & 8,20 & $-0,57$ & \\
\hline D30 & COELCE & 7,77 & 1,88 & $24 \%$ & 7,44 & 8.50 & $-1,06$ & \\
\hline D45 & ELEKTRO & 7,54 & 1,13 & $15 \%$ & 7,75 & 7,20 & 0,55 & \\
\hline D29 & COELBA & 7,40 & 2,03 & $27 \%$ & 7,00 & 8,20 & $-1,20$ & \\
\hline D16 & CEMAR & 7,27 & 2,31 & $32 \%$ & 6,70 & 8,40 & $-1,70$ & \\
\hline D01 & AES SUL & 7,13 & 1,19 & $17 \%$ & 6,90 & 7,60 & $-0,70$ & \\
\hline D04 & BANDEIRANTE & 7,07 & 1,14 & $16 \%$ & 7,00 & 7,25 & $-0,25$ & \\
\hline D18 & CEMIG & 7,00 & 1,20 & $17 \%$ & 7,20 & 6,60 & 0,60 & \\
\hline D48 & ELETROPAULO & 7,00 & 1,31 & $19 \%$ & 7,00 & 7,00 & 0,00 & \\
\hline D33 & COSERN & 7,00 & 2,09 & $30 \%$ & 6,88 & 7,25 & $-0,38$ & \\
\hline D50 & EMG (ENERGIA DE MINAS GERAIS) & 6,86 & 2,14 & $31 \%$ & 6,78 & 7,00 & $-0,22$ & \\
\hline D15 & CELTINS & 6,85 & 1,95 & $29 \%$ & 6,56 & 7,50 & $-0,94$ & \\
\hline D03 & AMPLA & 6,54 & 1,56 & $24 \%$ & 6,56 & 6,50 & 0,06 & \\
\hline D60 & LIGHT & 6,46 & 1,61 & $25 \%$ & 6,89 & 5,50 & 1,39 & \\
\hline D51 & ENERSUL & 6,38 & 1,19 & $19 \%$ & 6,11 & 7,00 & $-0,89$ & \\
\hline D14 & CELPE & 6,38 & 2,18 & $34 \%$ & 6,00 & 7,25 & $-1,25$ & \\
\hline D54 & ESCELSA & 6,38 & 2,18 & $34 \%$ & 6,00 & 7,25 & $-1,25$ & \\
\hline D53 & ENE. PARAÍBA & 6,33 & 2,31 & $36 \%$ & 6,38 & 6,25 & 0,13 & \\
\hline D55 & ENE. SERGIPE & 6,25 & 2,34 & $37 \%$ & 6,25 & 6,25 & 0,00 & \\
\hline D32 & COPEL & 6,14 & 0,77 & $13 \%$ & 6,10 & 6,25 & $-0,15$ & \\
\hline D17 & CEMAT & 5,77 & 1,74 & $30 \%$ & 5,56 & 6,25 & $-0,69$ & \\
\hline D11 & CELESC & 5,36 & 1,55 & $29 \%$ & 5,00 & 6,25 & $-1,25$ & \\
\hline D19 & CEPISA & 5,00 & 1,22 & $24 \%$ & 5,00 & 5,00 & 0,00 & \\
\hline D10 & CEEE & 4,93 & 1,73 & $35 \%$ & 4,60 & 5,75 & $-1,15$ & \\
\hline D13 & CELPA & 4,92 & 1,12 & $23 \%$ & 4,88 & 5,00 & $-0,13$ & \\
\hline D20 & CERON & 4,85 & 1,72 & $36 \%$ & 4,89 & 4,75 & 0,14 & \\
\hline D09 & CEB & 4,80 & 1,32 & $28 \%$ & 5,00 & 4,40 & 0,60 & \\
\hline D12 & CELG & 4,71 & 1,59 & $34 \%$ & 4,60 & 5,00 & $-0,40$ & \\
\hline D08 & CEAL & 4,31 & 1,11 & $26 \%$ & 4,33 & 4,25 & 0,08 & \\
\hline D46 & ELETROACRE & 4,09 & 1,97 & $48 \%$ & 4,00 & 4,25 & $-0,25$ & \\
\hline D02 & AME & 3,64 & 1,45 & $40 \%$ & 3,44 & 4,00 & $-0,56$ & \\
\hline
\end{tabular}

As colunas Média Especialistas, Desvio Padrão e Coeficiente de Variação consolidam as respostas de todos os especialistas, enquanto que a coluna Média Distribuidoras consolida as respostas de funcionários que formam o corpo técnico das empresas de distribuição. A coluna Média Consultores consolida as respostas de profissionais sem vínculo com empresas de distribuição. A coluna Dif Distrib. e Consult. consolida a diferença entre a coluna Média Distribuidoras e a coluna Média Consultores.

A Tabela 5 revela que algumas DMUs despertam percepções muito diversificadas no público de especialistas como um todo. É o caso da AME (coeficiente de variação igual a 40\%) e da ELETROACRE (48\%) Mas, de uma forma geral, os desvios relativos à média atingiram $27 \%$ do valor desta, o que pode ser considerado aceitável.

A análise da Tabela 5 também revela que algumas DMUs são consideradas mais eficientes por técnicos das empresas do setor do que por profissionais externos. O maior exemplo disso é a LIGHT. Já em outras empresas, como a CEMAR, ocorre o oposto. As diferenças, que não são tão significativas em termos absolutos, também 
não são significativas em termos comparativos. A correlação de Spearman entre as ordens das médias de técnicos e profissionais externos é de 0,88 .

\section{Tabela 6}

Ordenamento das DMUs conforme pesquisa com especialistas

\begin{tabular}{|c|c|c|c|c|c|c|}
\hline Pos & Ordenação por Especialistas & Média Esp. & Ordenação por Distribuidoras & Média Dis. & Ordenação por Consultores & Média Con. \\
\hline 1 & RGE & 8,4 & RGE & 8,2 & CPFL PAULISTA & 8,8 \\
\hline 2 & CPFL PAULISTA & 8,3 & CPFL PAULISTA & 8,1 & RGE & 8,6 \\
\hline 3 & PIRATININGA & 7,8 & ELEKTRO & 7,8 & COELCE & 8,5 \\
\hline 4 & COELCE & 7,8 & PIRATININGA & 7,6 & CEMAR & 8,4 \\
\hline 5 & ELEKTRO & 7,5 & COELCE & 7,4 & COELBA & 8,2 \\
\hline 6 & COELBA & 7,4 & CEMIG & 7,2 & PIRATININGA & 8,2 \\
\hline 7 & CEMAR & 7,3 & COELBA & 7,0 & AES SUL & 7,6 \\
\hline 8 & AES SUL & 7,1 & BANDEIRANTE & 7,0 & CELTINS & 7,5 \\
\hline 9 & BANDEIRANTE & 7,1 & ELETROPAULO & 7,0 & BANDEIRANTE & 7,3 \\
\hline 10 & CEMIG & 7,0 & AES SUL & 6,9 & CELPE & 7,3 \\
\hline 11 & ELETROPAULO & 7,0 & LIGHT & 6,9 & COSERN & 7,3 \\
\hline 12 & COSERN & 7,0 & COSERN & 6,9 & ESCELSA & 7,3 \\
\hline 13 & EMG (ENERGIA DE MINAS GERAIS) & 6,9 & EMG (ENERGIA DE MINAS GERAIS) & 6,8 & ELEKTRO & 7,2 \\
\hline 14 & CELTINS & 6,8 & CEMAR & 6,7 & ELETROPAULO & 7,0 \\
\hline 15 & AMPLA & 6,5 & CELTINS & 6,6 & EMG (ENERGIA DE MINAS GERAIS) & 7,0 \\
\hline 16 & LIGHT & 6,5 & AMPLA & 6,6 & ENERSUL & 7,0 \\
\hline 17 & ENERSUL & 6,4 & ENE. PARAÍBA & 6,4 & CEMIG & 6,6 \\
\hline 18 & CELPE & 6,4 & ENE. SERGIPE & 6,3 & AMPLA & 6,5 \\
\hline 19 & ESCELSA & 6,4 & ENERSUL & 6,1 & CELESC & 6,3 \\
\hline 20 & ENE. PARAÍBA & 6,3 & COPEL & 6,1 & CEMAT & 6,3 \\
\hline 21 & ENE. SERGIPE & 6,3 & CELPE & 6,0 & COPEL & 6,3 \\
\hline 22 & COPEL & 6,1 & ESCELSA & 6,0 & ENE. PARAÍBA & 6,3 \\
\hline 23 & CEMAT & 5,8 & CEMAT & 5,6 & ENE. SERGIPE & 6,3 \\
\hline 24 & CELESC & 5,4 & CELESC & 5,0 & CEEE & 5,8 \\
\hline 25 & CEPISA & 5,0 & CEPISA & 5,0 & LIGHT & 5,5 \\
\hline 26 & CEEE & 4,9 & CEB & 5,0 & CELG & 5,0 \\
\hline 27 & CELPA & 4,9 & CERON & 4,9 & CELPA & 5,0 \\
\hline 28 & CERON & 4,8 & CELPA & 4,9 & CEPISA & 5,0 \\
\hline 29 & CEB & 4,8 & CEEE & 4,6 & CERON & 4,8 \\
\hline 30 & CELG & 4,7 & CELG & 4,6 & CEB & 4,4 \\
\hline 31 & CEAL & 4,3 & CEAL & 4,3 & CEAL & 4,3 \\
\hline 32 & ELETROACRE & 4,1 & ELETROACRE & 4,0 & ELETROACRE & 4,3 \\
\hline 33 & AME & 3,6 & AME & 3,4 & AME & 4,0 \\
\hline
\end{tabular}

*Obs.: Pos = posição das DMUs, em ordem decrescente de eficiência conforme os especialistas.

A Tabela 6 comprova a alta correlação supracitada: os ordenamentos produzidos pelos dois segmentos de especialistas se assemelham, assim como a distância entre média de eficiência de duas DMUs quaisquer tem quase o mesmo valor nominal em ambos os ordenamentos, o que consolida a coleta e sugere que, de um modo geral, técnicos do setor e profissionais externos têm uma visão muito parecida sobre a eficiência das companhias.

O ordenamento apresentado na coluna Ordenação de Especialistas é um parâmetro a ser seguido pelos modelos que busquem representar a eficiência do setor. Assim sendo, modelos DEA com este objetivo devem ter alta correlação com os dados do resultado da pesquisa com especialistas. 


\subsection{Resultados da triangulação}

A triangulação foi utilizada para identificar se os modelos DEA propostos refletem a visão dos especialistas no setor. A Tabela 7 apresenta os primeiros resultados nesse sentido.

\section{Tabela 7}

Análise de correlação de Spearman dos modelos DEA com os resultados da Pesquisa com Especialistas

\begin{tabular}{|c|c|c|c|c|c|c|c|}
\hline Modelo & Correl. Especialistas & Correl. Distribuidoras & Correl. Consultores & Modelo & Correl. Especialistas & Correl. Distribuidoras & Correl. Consultores \\
\hline 1 & 0,802 & 0,787 & 0,751 & 24 & 0,832 & 0,813 & 0,790 \\
\hline 2 & 0,869 & 0,885 & 0,755 & 25 & 0,538 & 0,494 & 0,577 \\
\hline 3 & 0,057 & 0,018 & 0,125 & 26 & 0,536 & 0,500 & 0,556 \\
\hline 4 & 0,509 & 0,488 & 0,505 & 27 & 0,567 & 0,517 & 0,606 \\
\hline 5 & 0,697 & 0,713 & 0,596 & 28 & 0,535 & 0,494 & 0,561 \\
\hline 6 & 0,748 & 0,751 & 0,670 & 29 & 0,798 & 0,762 & 0,791 \\
\hline 7 & 0,807 & 0,813 & 0,717 & 30 & 0,748 & 0,740 & 0,695 \\
\hline 8 & 0,805 & 0,821 & 0,699 & 31 & 0,156 & 0,156 & 0,147 \\
\hline 9 & 0,790 & 0,769 & 0,751 & 32 & 0,467 & 0,479 & 0,406 \\
\hline 10 & 0,844 & 0,828 & 0,793 & 33 & 0,454 & 0,428 & 0,466 \\
\hline 11 & 0,755 & 0,761 & 0,668 & 34 & 0,796 & 0,814 & 0,689 \\
\hline 12 & 0,336 & 0,351 & 0,271 & 35 & 0,800 & 0,795 & 0,726 \\
\hline 13 & 0,806 & 0,784 & 0,772 & 36 & 0,850 & 0,810 & 0,843 \\
\hline 14 & 0,846 & 0,830 & 0,793 & 37 & 0,832 & 0,794 & 0,822 \\
\hline 15 & 0,801 & 0,787 & 0,748 & 38 & 0,913 & 0,872 & 0,907 \\
\hline 16 & 0,781 & 0,766 & 0,731 & 39 & 0,873 & 0,828 & 0,878 \\
\hline 17 & $-0,054$ & $-0,021$ & $-0,116$ & 40 & 0,912 & 0,870 & 0,907 \\
\hline 18 & 0,762 & 0,791 & 0,635 & 41 & 0,862 & 0,814 & 0,874 \\
\hline 19 & 0,675 & 0,697 & 0,569 & 42 & 0,909 & 0,886 & 0,867 \\
\hline 20 & 0,551 & 0,542 & 0,513 & 43 & 0,913 & 0,891 & 0,868 \\
\hline 21 & 0,595 & 0,552 & 0,625 & 44 & 0,870 & 0,843 & 0,838 \\
\hline 22 & 0,615 & 0,589 & 0,615 & 45 & 0,906 & 0,884 & 0,863 \\
\hline 23 & 0,826 & 0,798 & 0,807 & & & & \\
\hline
\end{tabular}

Observações: (1) A nomenclatura das colunas é análoga à utilizada na Tabela 8. (2) A seta indica o modelo com a maior correlação. (3) Foram usadas 3 casas decimais excepcionalmente, para ser possível diferenciar melhor a performance de cada modelo.

A Tabela 7 aponta o Modelo 43, proposto por Bogetoft e Lopes (2015) como contribuição à Nota Técnica № 407/2014 da ANEEL, como o modelo que melhor exprime a visão dos especialistas como um todo. Esse modelo também se destaca como o que melhor exprime a visão dos técnicos das distribuidoras, e tem bom desempenho no objetivo de exprimir a visão dos profissionais externos. Entretanto, o modelo mais assertivo para representar os consultores é o Modelo 40, que corresponde a um dos modelos propostos pela ANEEL na Nota Técnica oㅜ192/2014. Esses modelos têm características em comum, como a inserção de variáveis que exprimem qualidade como produtos negativos; o uso de rede, mercado ponderado e 
consumidores como produtos - ainda que segregados de formas diferentes - e a utilização de OPEX com correção de salários como insumo único. Aparentemente, essas características vão se consolidando como a melhor opção para retratar o setor elétrico nacional.

\subsubsection{Evolução dos modelos da ANEEL}

Foram analisados os modelos da ANEEL presentes nas Notas Técnicas n294/2011, no 192/2014 e o 407/2014. O objetivo dessa análise foi comparar os escores produzidos pelos modelos dessas notas técnicas com os resultados da pesquisa com especialistas.

\section{Figura 3}

Correlação da visão dos especialistas com a média dos escores de eficiência das empresas dos seis modelos da referida NT.

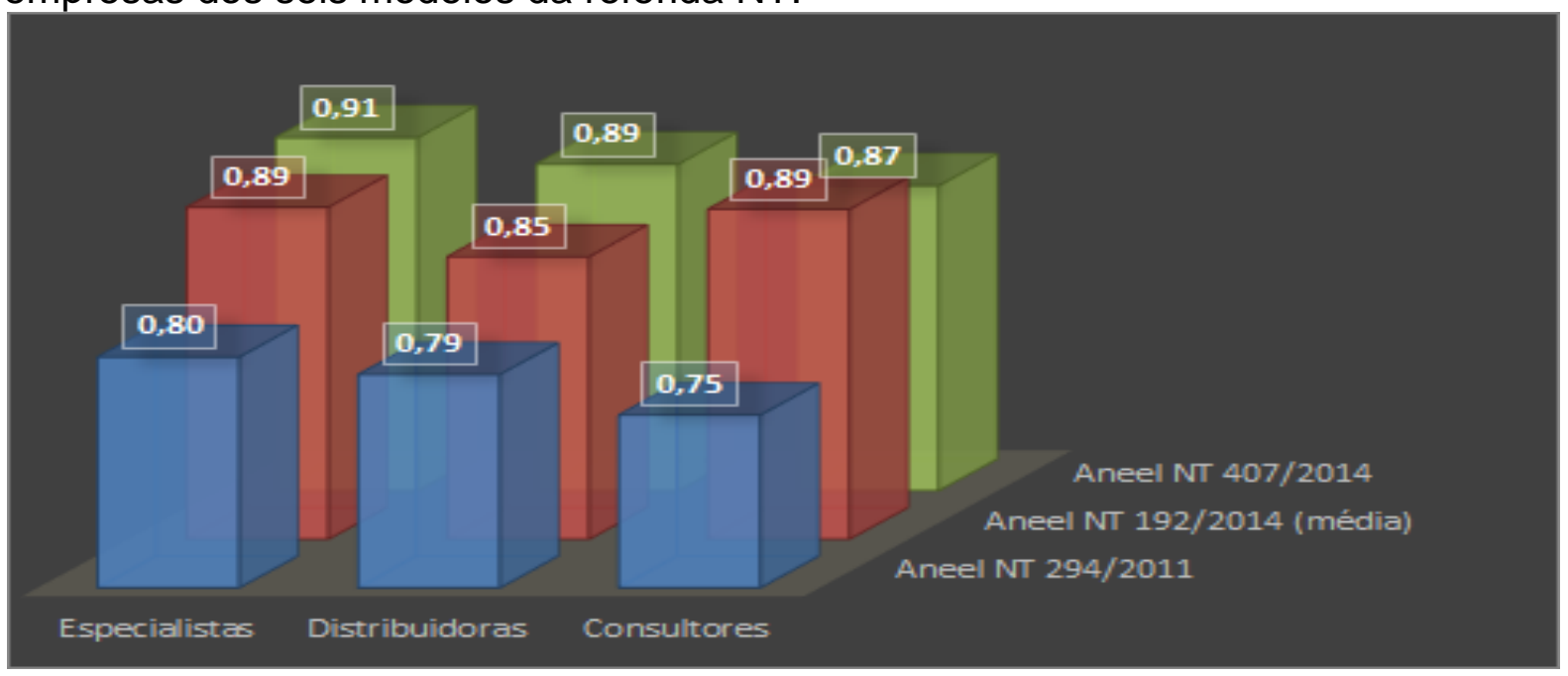

A Figura revela que, a cada nova Nota Técnica, a ANEEL tem obtido maior êxito em refletir a visão dos especialistas em geral. A Correlação de Spearman entre a visão dos especialistas e os modelos da ANEEL saltou de 0,8 a 0,91.

O mesmo fenômeno pode ser observado quando se analisa apenas a visão dos técnicos das distribuidoras. Contudo, ao analisar a visão do segmento de profissionais externos, a NT no 407/2014 representou um retrocesso para a nossa análise com relação à NT no 192/2014 como um todo. Ressalta-se que a NT no 192/2014 apresentou seis modelos, enquanto a NT no 407/2014 apresentou apenas um como solução. 
Também foi realizado o mesmo tipo de análise com as contribuições de Banker (2011) à Audiência Pública 040/2010 e de Bogetoft e Lopes (2015) à Audiência Pública 023/2015. O resultado está descrito na 8.

\section{Tabela 8}

Análise das Contribuições de Banker (2011), Bogetoft e Lopes (2015) e Notas Técnicas da ANEEL que as precederam

\begin{tabular}{llll|}
\hline Modelos & Especialistas & Distribuidoras & Consultores \\
\hline Aneel NT 294/2011 & 0,802 & 0,787 & 0,751 \\
\hline Banker & 0,869 & 0,885 & 0,755 \\
\hline Aneel NT 407/2014 & 0,909 & 0,886 & 0,867 \\
\hline Bogetoft \& Lopes Modelo B & 0,913 & 0,891 & 0,868 \\
\hline Bogetoft \& Lopes Modelo C & 0,870 & 0,843 & 0,838 \\
\hline Bogetoft \& Lopes Modelo D & 0,906 & 0,884 & 0,863 \\
\hline
\end{tabular}

Conforme a Tabela 8, é possível perceber que os modelos propostos por Banker (2011) e Bogetoft e Lopes (2015) têm trazido avanços nos esforços para se refletir a realidade do setor de distribuição. Assim como o modelo proposto por Banker (2011) apresentou resultados com maior correlação com a pesquisa com especialistas em todos os segmentos possíveis, em comparação com a NT no 294/2011, o mesmo ocorreu com o modelo B proposto por Bogetoft e Lopes (2015), desta vez tendo a NT № 407/2014 como comparação.

\section{Conclusões}

Neste artigo buscou-se identificar as modelagens DEA para o setor da distribuição de energia elétrica brasileira que melhor representam a visão de especialistas sobre a eficiência das empresas do setor.

A execução da pesquisa deste trabalho forneceu insumos para se concluir que a metodologia proposta se apresenta como um rico caminho para validar resultados obtidos através do uso de técnicas de benchmarking, sobretudo Data Envelopment Analysis- DEA. Por meio desta validação, concluiu-se que a visão de especialistas que estudam o setor de distribuição ou nele trabalham sobre a eficiência das 
empresas do setor pode ser refletida com sucesso utilizando-se alguns modelos estudados.

A visão dos técnicos que trabalham nas empresas do setor de distribuição sobre a eficiência destas é muito próxima da visão dos profissionais que estudam o setor, sendo eles pesquisadores de DEA ou consultores que atuam no setor. Abordando os especialistas como um todo, conclui-se que a base do modelo DEA proposto pela ANEEL para identificar a eficiência das empresas do setor de distribuição representada pela seleção de OPEX como insumo; rede, mercado e consumidores como produtos; NDRS como regime de escala - é uma base sólida e está no caminho certo.

A ANEEL vem progressivamente aprimorando seus modelos em torno da base supracitada e obtendo resultados mais apurados para representar a eficiência do setor de distribuição brasileiro. As contribuições de Banker (2011) e Bogetoft e Lopes (2015), realizadas através do Núcleo de Eficiência, Sustentabilidade e Produtividade (NESP) da Faculdade de Ciências Econômicas da UFMG, em geral, oferecem soluções ainda mais robustas para refletir a visão dos especialistas, contribuindo para o desenvolvimento da regulação no Brasil.

\section{Referências}

Associação Brasileira de Distribuidoras de Energia Elétrica. A distribuição de energia.

Disponível em: <http://www.abradee.com.br/setor-de-distribuicao/a-distribuicaode-energia>. Acesso em 26 de Abril de 2018.

Agência Nacional de Energia Elétrica (2011). Nota Técnica no 294/2011-SRE/ANEEL.

Disponível em: < http://www.aneel.gov.br/ >. Acesso em: 26 de Abril de 2018.

Agência Nacional de Energia Elétrica (2014). Nota Técnica no 192/2014-SRE/ANEEL.

Disponível em: < http://www.aneel.gov.br/ >. Acesso em: 26 de Abril de 2018.

Agência Nacional de Energia Elétrica (2014). Nota Técnica no 407/2014-SRE/ANEEL.

Disponível em: < http://www.aneel.gov.br/ >. Acesso em: 26 de Abril de 2018.

Agência Nacional de Energia Elétrica (2015). Nota Técnica no 66/2015-SRE/ANEEL. Disponível em: < http://www.aneel.gov.br/ >. Acesso em: 26 de Abril de 2018.

Agrell, P. J., \& Bogetoft, P. (2016). Regulatory benchmarking: Models, analyses and applications. DEA J (forthcoming). 
Banker, R. D., Charnes, A., \& Cooper, W. W. (1984). Some models for estimating technical and scale inefficiencies in data envelopment analysis. Management science, 30(9), 1078-1092.

Banker, R. (2011). Report on ANEEL's Proposal for Electricity Distribution Tariff Regulation Technical Note No. 101/2011. Disponível em: <http://www.aneel.gov.br/aplicacoes/audiencia/arquivo/2010/040/contribuicao/raji v_banker_and_ana_lopes_report.pdf>. Acesso em: 26 de Abril de 2018.

Bobde, S. M., \& Tanaka, M. (2018). Efficiency evaluation of electricity distribution utilities in India: A two-stage DEA with bootstrap estimation. Journal of the Operational Research Society, 1-12.

Bogetoft, P., \& Lopes, A.L.M. (2015). Comments on the Brazilian benchmarking model for energy distribution regulation fourth cycle of tariff review-Technical Note 407/2014. Disponível em: <http://www.aneel.gov.br/>. Acesso em: 26 de Abril de 2018.

Bougnol, M., \& Dula, J. H. (2006). Validating DEA as a ranking tool: An application of DEA to assess performance in higher education. Annals of Operations Research, 145(1), 339-365.

Calôba, G. M., \& Lins, M. P. E. (2005). Análise da Eficiência das Distribuidoras de Gás Natural Brasileiras Utilizando Análise Envoltória de Dados. In: SIMPÓSIO BRASILEIRO DE PESQUISA OPERACIONAL, 37, Gramado.

Charnes, A., Cooper, W. W., Golany, B., Seiford, L., \& Stutz, J. (1985). Foundations of data envelopment analysis for Pareto-Koopmans efficient empirical production functions. Journal of econometrics, 30(1-2), 91-107.

Charnes, A., Cooper, W. W., \& Rhodes, E. (1978) Measuring the efficiency of decision making units. European journal of operational research, 2(6), 429-444.

Cohen, J. (1992). Statistical power analysis. Current Directions in Psychological Science, 1(3), 98-101.

Cook, W. D., Ruiz, J. L., Sirvent, I., \& Zhu, J. (2017). Within-group common benchmarking using DEA. European Journal of Operational Research, 256(3), 901-910.

Corton, M. L., Zimmermann, A., \& Phillips, M. A. (2016). The low cost of quality improvements in the electricity distribution sector of Brazil. Energy Policy, 97, 485493.

Efron, B., \& Tibshirani, R. J. (1994). An introduction to the bootstrap. CRC press.

Farrel, M. J. (1957). The measurement of productive efficiency. Journal of the Royal Statistical Society, Series A (General), 120(3), 253-290. 
Giannakis, D., Jamasb, T., \& Pollitt, M. (2005). Benchmarking and incentive regulation of quality of service: an application to the UK electricity distribution networks. Energy Policy, 33(17), 2256-2271.

Growitsch, C., Jamasb, T., \& Pollitt, M. (2009). Quality of service, efficiency and scale in network industries: an analysis of European electricity distribution. Applied Economics, 41(20), 2555-2570.

Growitsch, C., Jamasb, T., \& Wetzel, H. (2010). Efficiency effects of quality of service and environmental factors: experience from Norwegian electricity distribution (No. 10/03). EWI Working Paper.

Jenkins, J. D., \& Pérez-Arriaga, I. J. (2017). Improved regulatory approaches for the remuneration of electricity distribution utilities with high penetrations of distributed energy resources. The Energy Journal, 38(3).

Lins, M. E., Lobo, M. S. D. C., Silva, A. C. M. D., Fiszman, R., \& Ribeiro, V. J. D. P. (2007). O uso da Análise Envoltória de Dados (DEA) para avaliação de hospitais universitários brasileiros. Ciência \& saúde coletiva, 12, 985-998.

LMDM Consultoria. Contribuições CP 011/2013. Disponível em: $<$ http://www.aneel.gov.br/aplicacoes/consulta_publica/documentos/LMDM\%20C ONSULTORIA\%20CP\%20011_2013.pdf>. Acesso em: 26 de Abril de 2018..

Lobo, M. S. D. C., Rodrigues, H. D. C., André, E. C. G., Azeredo, J. A. D., \& Lins, M. P. E. (2016). Dynamic network data envelopment analysis for university hospitals evaluation. Revista de saude publica, 50 .

Lugoboni, L. F., de Toledo Paulino, A., Zittei, M. V. M., \& da Silva Pereira, R. (2015). Importância da sustentabilidade para as empresas do setor de energia elétrica: utilização de relatório de sustentabilidade com base no GRI. Revista Metropolitana de Sustentabilidade (ISSN 2318-3233), 5(3), 04-25.

Mello, J. C. C. B. S., Meza, L. A., Gomes, E. G., Serapião, B. P., \& Lins, M. P. E. (2003). Análise de envoltória de dados no estudo da eficiência e dos benchmarks para companhias aéreas brasileiras. Pesquisa Operacional, 23(2), 325-345.

Mukaka, M. M. (2012). A guide to appropriate use of Correlation coefficient in medical research. Malawi Medical Journal, 24(3), 69-71.

Peano, C. R. (2005). Regulação tarifária do setor de distribuição de energia elétrica no Brasil: uma análise da metodologia de revisão tarifária adotada pela Aneel. São Paulo.

Pereira, N. A., \& Tavares, M. (2017). Efficiency of major producing regions of sugar cane through Data Envelopment Analysis (DEA). CEP, 38, 100.

Péres, V. M., Campos, M. V., \& Liang, T. L. S. (2015). Smart Grid: A Chance for Brazilian Electric Distribution. Journal INNOVER, 1(4), 106-118. 
Périco, A. E., Santana, N. B., \& Rebelatto, D. A. D. N. (2017). Efficiency of Brazilian international airports: applying the bootstrap data envelopment analysis. Gestão \& Produção, 24(2), 370-381.

Pessanha, J. F. M., Souza, R. C., \& Laurencel, L. D. C. (2007). Um modelo de análise envoltória de dados para o estabelecimento de metas de continuidade do fornecimento de energia elétrica. Pesquisa Operacional, 27(1), 51-83.

Pires, J. C., \& Piccinini, M. S. (1999). A regulação dos setores de infra-estrutura no Brasil. A economia brasileira nos anos 90, 217-260.

Rødseth, K. L. (2017). Environmental regulations and allocative efficiency: application to coal-to-gas substitution in the US electricity sector. Journal of Productivity Analysis, 47(2), 129-142.

Salgado, L. H., \& Motta, R. S. (2005). Marcos regulatórios no Brasil: o que foi feito e o que falta fazer. Rio de Janeiro: Ipea.

Saurin, V., Lopes, A. L. M., \& da Costa Júnior, N. C. (2010). Eficiência e valor: uma abordagem com base na análise envoltória de dados (DEA) aplicada às empresas do setor elétrico no Brasil. Revista de Economia e Administração, 9(2).

Senra, L. F. A. D. C., Nanci, L. C., Mello, J. C. C. B. S., \& Meza, L. A. (2007). Estudo sobre métodos de seleção de variáveis em DEA. Pesquisa Operacional, 27(2), 191-207.

Siciliano, A. (2005). Regulação incentivada: simplificação ou complicação na supervisão das concessionárias de eletricidade. Revista do BNDES, 12(23), 243266.

Sollero, M. K. V., \& Lins, M. P. E. (2004). Avaliação de eficiência de distribuidoras de energia elétrica através da análise envoltória de dados com restrições aos pesos. XXXVI Simpósio Brasileiro de Pesquisa Operacional, São João del Rei.

Zanini, A. (2004). Regulação econômica no setor elétrico brasileiro: uma metodologia para definição de fronteiras de eficiência e cálculo do fator $X$ para empresas distribuidoras de energia elétrica. Pontifícia Universidade Católica do Rio de Janeiro, Rio de Janeiro. 
APÊNDICE I - CONFIGURAÇÕES DOS MODELOS DEA AVALIADOS

\begin{tabular}{|c|c|c|c|c|c|c|}
\hline MODELO & INSUMOS & PRODUTOS & $\begin{array}{l}\text { RETORNO } \\
\text { DE } \\
\text { ESCALA }\end{array}$ & $\begin{array}{l}\text { QTD DE } \\
\text { VARIÁVEIS }\end{array}$ & $\begin{array}{l}\text { QTD DE } \\
\text { DMUS }\end{array}$ & FONTE \\
\hline 1 & OPEX & $\begin{array}{l}\text { MERCADO PONDERADO, } \\
\text { NÚMERO DE } \\
\text { CONSUMIDORES, REDE } \\
\text { TOTAL }\end{array}$ & NDRS & 4 & 203 & ANEEL, NT 294/2011 \\
\hline 2 & OPEX & $\begin{array}{l}\text { MERCADO PONDERADO, } \\
\text { NÚMERO DE } \\
\text { CONSUMIDORES, REDE } \\
\text { TOTAL }\end{array}$ & VRS & 4 & 203 & $\begin{array}{l}\text { BANKER, LOPES, } \\
\text { CONTRIBUIÇÃO } 2011\end{array}$ \\
\hline 3 & OPEX, CAPEX & $\begin{array}{l}\text { MERCADO PONDERADO, } \\
\text { NÚMERO DE } \\
\text { CONSUMIDORES, REDE } \\
\text { TOTAL }\end{array}$ & NDRS & 5 & 203 & $\begin{array}{l}\text { DESMEMBRAMENTO } \\
\text { MODELO } 1\end{array}$ \\
\hline 4 & TOTEX & $\begin{array}{l}\text { MERCADO PONDERADO, } \\
\text { NÚMERO DE } \\
\text { CONSUMIDORES, REDE } \\
\text { TOTAL }\end{array}$ & NDRS & 4 & 203 & $\begin{array}{l}\text { HÍBRIDO } \\
\text { GROWITSCH (2009) E } \\
\text { ANEEL }\end{array}$ \\
\hline 5 & $\begin{array}{l}\text { OPEX AJUSTADO } \\
\text { SALÁRIO }\end{array}$ & $\begin{array}{l}\text { MERCADO PONDERADO, } \\
\text { NÚMERO DE } \\
\text { CONSUMIDORES, REDE } \\
\text { TOTAL }\end{array}$ & NDRS & 4 & 203 & SUGESTÃO DO NESP \\
\hline 6 & OPEX & $\begin{array}{l}\text { MERCADO PONDERADO, } \\
\text { NÚMERO DE } \\
\text { CONSUMIDORES, REDE } \\
\text { URBANA, REDE RURAL }\end{array}$ & NDRS & 5 & 203 & $\begin{array}{l}\text { DESMEMBRAMENTO } \\
\text { MODELO } 1\end{array}$ \\
\hline 7 & OPEX & $\begin{array}{l}\text { MERCADO ALTA TENSÃO } \\
\text { - AT, MERCADO MÉDIA } \\
\text { TENSÃO - MT, MERCADO } \\
\text { BAIXA TENSÃO - BT, } \\
\text { NÚMERO DE } \\
\text { CONSUMIDORES, REDE } \\
\text { TOTAL }\end{array}$ & NDRS & 6 & 203 & $\begin{array}{l}\text { DESMEMBRAMENTO } \\
\text { MODELO } 1\end{array}$ \\
\hline 8 & OPEX & $\begin{array}{l}\text { MERCADO PONDERADO, } \\
\text { NÚMERO DE } \\
\text { CONSUMIDORES, REDE } \\
\text { TOTAL, NÚMERO DE } \\
\text { TRANSFORMADORES }\end{array}$ & NDRS & 5 & 203 & SUGESTÃO DO NESP \\
\hline 9 & OPEX & $\begin{array}{l}\text { MERCADO PONDERADO, } \\
\text { NÚMERO DE } \\
\text { CONSUMIDORES, } \\
\text { EXTENSÃO DE REDE AT, } \\
\text { MT, BT }\end{array}$ & NDRS & 6 & 203 & $\begin{array}{l}\text { DESMEMBRAMENTO } \\
\text { MODELO } 1\end{array}$ \\
\hline 10 & $\begin{array}{l}\text { OPEX + ENERGIA NÃO } \\
\text { SUPRIDA (ENCARGOS } \\
\text { DO USO DA } \\
\text { DISTRIBUIÇÃO * № DE } \\
\text { CONSUMIDORES * SOMA } \\
\text { DAS HORAS } \\
\text { INDISPONÍVEIS), TEMPO } \\
\text { TOTAL DAS } \\
\text { INTERRUPC̃ÕES } \\
\text { (DEC*NUC (№ DE } \\
\text { CONSUMIDORES)) }\end{array}$ & $\begin{array}{l}\text { MERCADO PONDERADO, } \\
\text { NÚMERO DE } \\
\text { CONSUMIDORES }\end{array}$ & NDRS & 4 & 203 & GROWITSCH (2010) \\
\hline 11 & OPEX & $\begin{array}{l}\text { MERCADO PONDERADO, } \\
\text { NÚMERO DE } \\
\text { CONSUMIDORES, REDE } \\
\text { TOTAL, TEMPO MÉDIO } \\
\text { DE DESLOCAMENTO - } \\
\text { TMD (DISTANCIA) }\end{array}$ & NDRS & 5 & 203 & SUGESTÃO DO NESP \\
\hline 12 & $\begin{array}{l}\text { EXTENSÃO DE REDE AT, } \\
\text { EXTENSÃO DE REDE MT, } \\
\text { EXTENSÃO DE REDE BT, } \\
\text { NÚMERO DE } \\
\text { TRANSFORMADORES, } \\
\text { PESSOAL, TERCEIROS, } \\
\text { MATERIAIS, OUTRAS } \\
\text { DESPESAS }\end{array}$ & $\begin{array}{l}\text { MERCADO PONDERADO, } \\
\text { NÚMERO DE } \\
\text { CONSUMIDORES }\end{array}$ & NDRS & 10 & 203 & SUGESTÃO DO NESP \\
\hline
\end{tabular}




\begin{tabular}{|c|c|c|c|c|c|c|c|}
\hline 13 & $\begin{array}{l}\text { OPEX, CUSTO DE } \\
\text { COMPENSAÇÃO }\end{array}$ & $\begin{array}{l}\text { MERCADO PONDERADO, } \\
\text { NÚMERO DE } \\
\text { CONSUMIDORES, REDE } \\
\text { TOTAL }\end{array}$ & NDRS & 5 & \multicolumn{2}{|c|}{203} & SUGESTÃO DO NESP \\
\hline 14 & $\begin{array}{l}\text { OPEX, TEMPO TOTAL } \\
\text { DAS INTERRUPÇÕES }\end{array}$ & $\begin{array}{l}\text { MERCADO PONDERADO, } \\
\text { NÚMERO DE } \\
\text { CONSUMIDORES }\end{array}$ & NDRS & 4 & \multicolumn{2}{|c|}{203} & GROWITSCH (2009) \\
\hline 15 & OPEX SEM TRIBUTOS & $\begin{array}{l}\text { MERCADO PONDERADO, } \\
\text { NÚMERO DE } \\
\text { CONSUMIDORES, REDE } \\
\text { TOTAL }\end{array}$ & NDRS & 4 & \multicolumn{2}{|c|}{203} & $\begin{array}{l}\text { RELATÓRIO PARA } \\
\text { ABRADEE, } \\
\text { MERCADOS / PSR }\end{array}$ \\
\hline 16 & OPEX & \multicolumn{2}{|c|}{$\begin{array}{l}\text { MERCADO PONDERADO, EXTENSÃO } \\
\text { DE REDE AT, MT, BT }\end{array}$} & NDRS & 5 & 203 & $\begin{array}{l}\text { ELABORADO PELO } \\
\text { AUTOR }\end{array}$ \\
\hline 17 & OPEX, CAPEX & \multicolumn{2}{|l|}{ MERCADO PONDERADO } & NDRS & 3 & 203 & $\begin{array}{l}\text { ELABORADO PELO } \\
\text { AUTOR }\end{array}$ \\
\hline 18 & OPEX & \multicolumn{2}{|c|}{$\begin{array}{l}\text { MERCADO PONDERADO, NÚMERO DE } \\
\text { TRANSFORMADORES }\end{array}$} & NDRS & 3 & 203 & $\begin{array}{l}\text { ELABORADO PELO } \\
\text { AUTOR }\end{array}$ \\
\hline 19 & OPEX & \multicolumn{2}{|c|}{$\begin{array}{l}\text { MERCADO PONDERADO, REDE } \\
\text { URBANA, REDE RURAL }\end{array}$} & NDRS & 4 & 203 & $\begin{array}{l}\text { ELABORADO PELO } \\
\text { AUTOR }\end{array}$ \\
\hline 20 & TOTEX & \multicolumn{2}{|c|}{$\begin{array}{l}\text { MERCADO PONDERADO, NÚMERO DE } \\
\text { CONSUMIDORES }\end{array}$} & NDRS & 3 & 203 & GROWITSCH (2009) \\
\hline 21 & TOTEX & \multicolumn{2}{|c|}{$\begin{array}{l}\text { ENERGIA ENTREGUE(MWH), NÚMERO } \\
\text { DE CONSUMIDORES, REDE TOTAL }\end{array}$} & CRS & 4 & 203 & $\begin{array}{l}\text { JAMASB, } \\
\text { POLLITT(2003) } \\
\text { GIANNAKIS ET ALL } \\
\text { (2005), ENERGY } \\
\text { POLICY, DEA-2VRS }\end{array}$ \\
\hline 22 & TOTEX & \multicolumn{2}{|c|}{$\begin{array}{l}\text { ENERGIA ENTREGUE(MWH), NÚMERO } \\
\text { DE CONSUMIDORES, REDE TOTAL }\end{array}$} & VRS & 4 & 203 & $\begin{array}{l}\text { JAMASB, } \\
\text { POLLITT(2003) } \\
\text { GIANNAKIS ET AL } \\
\text { (2005), ENERGY } \\
\text { POLICY, DEA-1VRS }\end{array}$ \\
\hline 23 & $\begin{array}{l}\text { OPEX, TEMPO TOTAL } \\
\text { DAS INTERRUPÇÕES }\end{array}$ & \multicolumn{2}{|c|}{$\begin{array}{l}\text { ENERGIA FATURADA(MWH), NÚMERO } \\
\text { DE CONSUMIDORES, REDE TOTAL }\end{array}$} & NRDS & 5 & 203 & $\begin{array}{l}\text { PSR/MERCADOS, } \\
\text { RELATÓRIO } 4 \text { - } \\
\text { ABRADEE }\end{array}$ \\
\hline 24 & $\begin{array}{l}\text { OPEX, ENERGIA NÃO } \\
\text { SUPRIDA }\end{array}$ & \multicolumn{2}{|c|}{$\begin{array}{l}\text { ENERGIA FATURADA(MWH), NÚMERO } \\
\text { DE CONSUMIDORES, REDE TOTAL }\end{array}$} & NDRS & 5 & 203 & $\begin{array}{l}\text { PSR/MERCADOS, } \\
\text { RELATÓRIO } 4 \text { - } \\
\text { ABRADEE }\end{array}$ \\
\hline 25 & $\begin{array}{l}\text { TOTEX, TEMPO TOTAL } \\
\text { DAS INTERRUPÇÕES }\end{array}$ & \multicolumn{2}{|c|}{$\begin{array}{l}\text { ENERGIA FATURADA(MWH), NÚMERO } \\
\text { DE CONSUMIDORES, REDE TOTAL }\end{array}$} & NRDS & 5 & 203 & $\begin{array}{l}\text { PSR/MERCADOS, } \\
\text { RELATÓRIO } 4 \text { - } \\
\text { ABRADEE }\end{array}$ \\
\hline 26 & $\begin{array}{l}\text { TOTEX, ENERGIA NÃO } \\
\text { SUPRIDA }\end{array}$ & \multicolumn{2}{|c|}{$\begin{array}{l}\text { ENERGIA FATURADA(MWH), NÚMERO } \\
\text { DE CONSUMIDORES, REDE TOTAL }\end{array}$} & NDRS & 5 & 203 & $\begin{array}{l}\text { PSR/MERCADOS, } \\
\text { RELATÓRIO } 4 \text { - } \\
\text { ABRADEE }\end{array}$ \\
\hline 27 & $\begin{array}{l}\text { TOTEX, NÚMERO DE } \\
\text { INTERRUPÇÕES } \\
\text { (FEC*NUC), TEMPO } \\
\text { TOTAL DAS } \\
\text { INTERRUPÇÕES }\end{array}$ & \multicolumn{2}{|c|}{$\begin{array}{l}\text { UNIDADES DE ENERGIA ENTREGUE, } \\
\text { NÚMERO DE CONSUMIDORES, REDE } \\
\text { TOTAL }\end{array}$} & CRS & 6 & 203 & $\begin{array}{l}\text { GIANNAKIS ET } \\
\text { ALL/ENERGY } \\
\text { POLICY, } 2005\end{array}$ \\
\hline 28 & $\begin{array}{l}\text { TOTEX, NÚMERO DE } \\
\text { INTERRUPÇÕES, TEMPO } \\
\text { TOTAL DAS } \\
\text { INTERRUPÇÕES }\end{array}$ & \multicolumn{2}{|c|}{$\begin{array}{l}\text { UNIDADES DE ENERGIA ENTREGUE, } \\
\text { NÚMERO DE CONSUMIDORES, REDE } \\
\text { TOTAL }\end{array}$} & VRS & 6 & 203 & $\begin{array}{l}\text { GIANNAKIS ET } \\
\text { ALL/ENERGY } \\
\text { POLICY, } 2005\end{array}$ \\
\hline 29 & OPEX & \multicolumn{2}{|c|}{$\begin{array}{l}\text { UNIDADES DE ENERGIA ENTREGUE, } \\
\text { NÚMERO DE CONSUMIDORES, REDE } \\
\text { TOTAL }\end{array}$} & CRS & 4 & 203 & $\begin{array}{l}\text { GIANNAKIS ET } \\
\text { ALL/ENERGY } \\
\text { POLICY, 2005, } \\
\text { REGULADOR } \\
\text { FINLÂNDIA }\end{array}$ \\
\hline 30 & OPEX & \multicolumn{2}{|c|}{$\begin{array}{l}\text { ENERGIA DISTRIBUIDA, EXTENSÃO DE } \\
\text { REDE SEGREGADA EM MÉDIA E BAIXA } \\
\text { VOLTAGEM E EM SUBTERRÂNEO OU } \\
\text { NÃO, NÚMERO DE } \\
\text { TRANSFORMADORES }\end{array}$} & NDRS & 4 & 203 & $\begin{array}{l}\text { REGULADOR } \\
\text { DINAMARCA }\end{array}$ \\
\hline
\end{tabular}
CONSUMIDORES, REDE TOTAL

MERCADO PONDERADO, NDRS

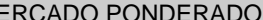
NÚMERO DE TOTAL

MERCADO PONDERADO, EXTENSÃO

MERCADO PONDERADO

MERCADO PONDERADO, NÚMERO DE

MERCADO PONDERADO, REDE

MERCADO PONDERADO, NÚMERO DE

ENERGIA ENTREGUE(MWH), NÚMERO

GIANNAKIS ET AL (2005), ENERGY

JAMASB,

POLLITT(2003)

GIANNAKIS ET AL

POLICY, DEA-1VRS RELATÓRIO 4 PSR/MERCADOS RELATORIO 4 -

PSR/MERCADOS ABRADEE

READOS,

PELATORIO

GIANNAKIS ET

ALL/ENERGY

\begin{tabular}{l|l|l|l|l}
\hline TOTAL DE & POLICY, 2005
\end{tabular}

UNIDADES DE ENERGIA ENTREGUE, NÚMERO DE CONSUMIDORES, REDE

GIANNAKIS E

ALL/ENERGY

UNIDADES DE ENERGIA ENTREGUE, NÚMERO DE CONSUMIDORES, REDE TOTAL

REGULADOR

FINLÂNDIA

REDE SEGREGADA EM MÉDIA E BAIXA

VETTAGEM E EM SUBTERRÂNEO OU

NÃO, NÚMERO DE 


\begin{tabular}{|c|c|c|c|c|c|c|}
\hline 31 & OPEX, CAPEX & $\begin{array}{l}\text { MERCADO PONDERADO, NÚMERO DE } \\
\text { TRANSFORMADORES, NÚMERO DE } \\
\text { CONSUMIDORES }\end{array}$ & NDRS & 5 & 203 & $\begin{array}{l}\text { ELABORADO PELO } \\
\text { AUTOR }\end{array}$ \\
\hline 32 & TOTEX & MERCADO AT MT BT, REDE TOTAL & NDRS & 3 & 203 & $\begin{array}{l}\text { ELABORADO PELO } \\
\text { AUTOR }\end{array}$ \\
\hline 33 & TOTEX & $\begin{array}{l}\text { MERCADO PONDERADO, REDE AT MT } \\
\text { BT }\end{array}$ & NDRS & 2 & 203 & $\begin{array}{l}\text { ELABORADO PELO } \\
\text { AUTOR }\end{array}$ \\
\hline 34 & OPEX & $\begin{array}{l}\text { MERCADO PONDERADO, NÚMERO DE } \\
\text { CONSUMIDORES, NÚMERO DE } \\
\text { TRANSFORMADORES }\end{array}$ & NDRS & 4 & 203 & $\begin{array}{l}\text { ELABORADO PELO } \\
\text { AUTOR }\end{array}$ \\
\hline 35 & OPEX & $\begin{array}{l}\text { MERCADO PONDERADO, NÚMERO DE } \\
\text { CONSUMIDORES, POTÊNCIA MVA } \\
\text { TRANSFORMADORES }\end{array}$ & NDRS & 4 & 203 & $\begin{array}{l}\text { ELABORADO PELO } \\
\text { AUTOR }\end{array}$ \\
\hline 36 & $\begin{array}{l}\text { OPEX COM CORREÇÃO } \\
\text { DE SALÁRIOS }\end{array}$ & $\begin{array}{l}\text { MERCADO PONDERADO, NÚMERO DE } \\
\text { CONSUMIDORES, REDE TOTAL }\end{array}$ & NDRS & 4 & 124 & $\begin{array}{l}\text { ANEEL, NT 192/2014, } \\
\text { MODELO } 1\end{array}$ \\
\hline 37 & $\begin{array}{l}\text { OPEX COM CORREÇÃO } \\
\text { DE SALÁRIOS }\end{array}$ & $\begin{array}{l}\text { MVA INSTALADO, NÚMERO DE } \\
\text { CONSUMIDORES, REDE TOTAL }\end{array}$ & NDRS & 4 & 124 & $\begin{array}{l}\text { ANEEL, NT 192/2014, } \\
\text { MODELO } 2\end{array}$ \\
\hline 38 & $\begin{array}{l}\text { OPEX COM CORREÇÃO } \\
\text { DE SALÁRIOS }\end{array}$ & $\begin{array}{l}\text { MERCADO PONDERADO, NÚMERO DE } \\
\text { CONSUMIDORES, REDE TOTAL, } \\
\text { PERDAS NÃO TÉCNICAS (PNT) - } \\
\text { NEGATIVO, ENDIST - NEGATIVO }\end{array}$ & NDRS & 6 & 124 & $\begin{array}{l}\text { ANEEL, NT 192/2014, } \\
\text { MODELO } 3\end{array}$ \\
\hline 39 & $\begin{array}{l}\text { OPEX COM CORREÇÃO } \\
\text { DE SALÁRIOS }\end{array}$ & $\begin{array}{l}\text { MVA INSTALADO, NÚMERO DE } \\
\text { CONSUMIDORES, REDE TOTAL, } \\
\text { PERDAS NÃO TÉCNICAS (PNT)- } \\
\text { NEGATIVO, ENDIST - NEGATIVO }\end{array}$ & NDRS & 6 & 124 & $\begin{array}{l}\text { ANEEL, NT 192/2014, } \\
\text { MODELO } 4\end{array}$ \\
\hline 40 & $\begin{array}{l}\text { OPEX COM CORREÇÃO } \\
\text { DE SALÁRIOS }\end{array}$ & $\begin{array}{l}\text { REDE ALTA E REDE DIST., MERCADO } \\
\text { PONDERADO, CONSUMIDORES } \\
\text { RURAIS E URBANOS, PERDAS NÃO } \\
\text { TÉCNICAS (PNT) - NEGATIVO, ENDIST - } \\
\text { NEGATIVO }\end{array}$ & NDRS & 6 & 124 & $\begin{array}{l}\text { ANEEL, NT 192/2014, } \\
\text { MODELO } 5\end{array}$ \\
\hline 41 & $\begin{array}{l}\text { OPEX COM CORREÇÃO } \\
\text { DE SALÁRIOS }\end{array}$ & $\begin{array}{l}\text { REDE ALTA E REDE DIST., MVA } \\
\text { INSTALADO, CONSUMIDORES RURAIS } \\
\text { E URBANOS, PERDAS NÃO TÉCNICAS } \\
\text { (PNT) - NEGATIVO, ENDIST - NEGATIVO }\end{array}$ & NDRS & 6 & 124 & $\begin{array}{l}\text { ANEEL, NT 192/2014, } \\
\text { MODELO } 6\end{array}$ \\
\hline 42 & $\begin{array}{l}\text { OPEX COM CORREÇÃO } \\
\text { DE SALÁRIOS }\end{array}$ & $\begin{array}{l}\text { REDE AT (A3, A2 E A1), REDE DE } \\
\text { DISTRIBUIÇÃO AÉREA (A3A, A4 E BT), } \\
\text { REDE DE DISTRIBUIÇÃO } \\
\text { SUBTERRÂNEA (AS), MERCADO } \\
\text { PONDERADO, NUUMERO DE } \\
\text { CONSUMIDORES, PERDAS NÃO } \\
\text { TÉCNICAS (PNT) - NEGATIVO, ENDIST - } \\
\text { NEGATIVO }\end{array}$ & NDRS & 8 & 61 & ANEEL, NT 407/2014 \\
\hline 43 & $\begin{array}{l}\text { OPEX COM CORREÇĀO } \\
\text { DE SALÁRIOS }\end{array}$ & $\begin{array}{l}\text { REDE AT (A3, A2 E A1), REDE DE } \\
\text { DISTRIBUIÇÂO AÉREA (A3A, A4 E BT), } \\
\text { REDE DE DISTRIBUIÇÃO } \\
\text { SUBTERRÂNEA (AS), MERCADO } \\
\text { PONDERADO, NÚMERO DE } \\
\text { CONSUMIDORES, PERDAS NÃO } \\
\text { TÉCNICAS (PNT) - NEGATIVO, ENDIST - } \\
\text { NEGATIVO, NÚMERO DE } \\
\text { TRANSFORMADORES }\end{array}$ & NDRS & 9 & 61 & $\begin{array}{l}\text { BOGETOFT, LOPES } \\
\text { (2015), MODELO B, } \\
\text { NESP - OFICIAL }\end{array}$ \\
\hline 44 & $\begin{array}{l}\text { OPEX COM CORREÇÃO } \\
\text { DE SALÁRIOS }\end{array}$ & $\begin{array}{l}\text { REDE AT (A3, A2 É A1), REDE DE } \\
\text { DISTRIBUIÇÂO AÉREA (A3A, A4 E BT), } \\
\text { REDE DE DISTRIBUIÇÃO } \\
\text { SUBTERRAANEA (AS), MERCADO } \\
\text { PONDERADO, NÚMERO DE } \\
\text { CONSUMIDORES, PERDAS NÃO } \\
\text { TÉCNICAS (PNT) - NEGATIVO, ENDIST - } \\
\text { NEGATIVO, NÚMERO DE } \\
\text { TRANSFORMADORES }\end{array}$ & NDRS & 9 & 61 & $\begin{array}{l}\text { BOGETOFT, LOPES } \\
\text { (2015), MODELO C, } \\
\text { NESP - OFICIAL }\end{array}$ \\
\hline
\end{tabular}




\begin{tabular}{|c|c|c|c|c|c|c|}
\hline 45 & $\begin{array}{l}\text { OPEX COM CORREÇÃO } \\
\text { DE SALÁRIOS }\end{array}$ & $\begin{array}{l}\text { REDE AT (A3, A2 E A1), REDE DE } \\
\text { DISTRIBUIÇÃO AÉREA (A3A, A4 E BT), } \\
\text { REDE DE DISTRIBUIÇÃO } \\
\text { SUBTERRÂNEA (AS), MERCADO } \\
\text { PONDERADO, NÚMERO DE } \\
\text { CONSUMIDORES, PERDAS NÃO } \\
\text { TÉCNICAS (PNT) - NEGATIVO, ENDIST - } \\
\text { NEGATIVO, NÚMERO DE } \\
\text { TRANSFORMADORES }\end{array}$ & NDRS & 9 & 61 & $\begin{array}{l}\text { BOGETOFT, LOPES } \\
\text { (2015), MODELO D, } \\
\text { NESP - OFICIAL }\end{array}$ \\
\hline
\end{tabular}

